Portland State University

PDXScholar

5-8-1996

\title{
La Representation de la Femme Aristocrate en Periode Post-revolutionnaire: Balzac Moraliste Chretien et Apologiste de la Passion
}

Isabelle Marie Renard

Portland State University

Follow this and additional works at: https://pdxscholar.library.pdx.edu/open_access_etds

Part of the French and Francophone Language and Literature Commons Let us know how access to this document benefits you.

Recommended Citation

Renard, Isabelle Marie, "La Representation de la Femme Aristocrate en Periode Post-revolutionnaire: Balzac Moraliste Chretien et Apologiste de la Passion" (1996). Dissertations and Theses. Paper 5144. https://doi.org/10.15760/etd.7020

This Thesis is brought to you for free and open access. It has been accepted for inclusion in Dissertations and Theses by an authorized administrator of PDXScholar. Please contact us if we can make this document more accessible: pdxscholar@pdx.edu. 


\section{THESIS APPROVAL}

The abstract and thesis of Isabelle Marie Renard for the Master of Arts in French were presented May 8, 1996, and accepted by the thesis committee and the department.

COMMITTEE APPROVALS:
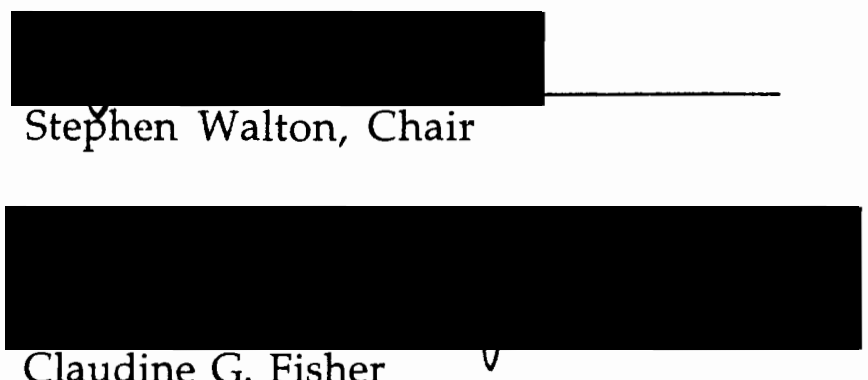

Claudine G. Fisher

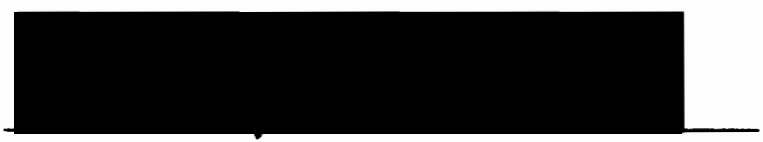

Eric Swenson

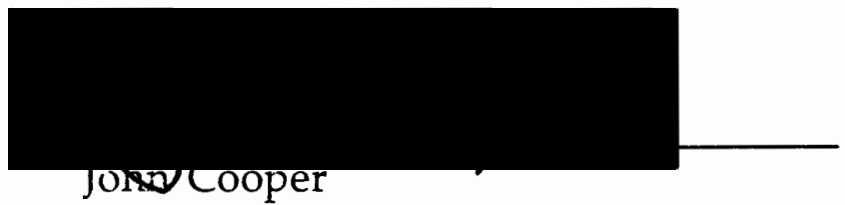

Representative of the Office of Graduate Studies

DEPARTMENT APPROVAL:

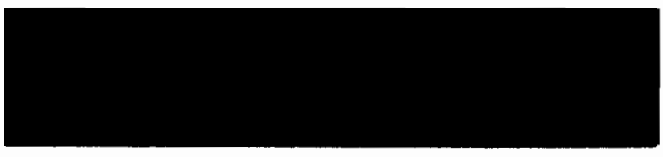

Louis Elteto, Chair

Department of Foreign Languages and Literatures

ACCEPTED FOR PORTLAND STATE UNIVERSITY BY THE LIBRARY by on

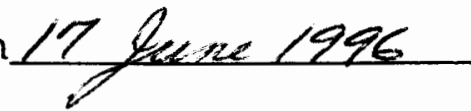




\section{ABSTRACT}

An abstract of the thesis of Isabelle Marie Renard for the Master of Arts in French presented May 8, 1996.

Title: La Représentation de la femme aristocrate en période post-révolutionnaire: Balzac moraliste chrétien et apologiste de la passion.

Honoré de Balzac appartient à cette génération de géants $\mathrm{du}$ romantisme flamboyant: politiquement et socialement, il est honorable bourgeois, se souvient des déceptions de l'époque déchue et prône par conséquent le culte du souvenir impérial, ainsi que celui de la passion. C'est pourquoi nous trouvons de constantes ambivalences dans la représentation de la femme du monde ainsi que des contradictions déroutantes quant à leur droit d'aimer. Balzac peint des êtres d'exception, mais, malgré la place et le rôle très important qu'il leur accorde, ces femmes subissent le droit de jugement ultime de l'auteur quand elles s'abandonnent à la passion; leur droit d'aimer semble dans un premier temps tout à fait légitime, puis il semble toujours que la morale chrétienne, tellement présente à l'époque de l'auteur et dans son esprit, l'emporte finalement.

Nous verrons, dans un premier chapitre, comment Balzac analysait la société comme un organisme animal, les espèces humaines comme des espèces naturelles. Son goût pour la physiologie se retrouve dans la représentation de son monde qui 
naît d'un don d'observation et d'imagination extraordinaire.

Dans un second chapitre, je tâcherai de présenter la vision critique que Balzac avait de son temps; c'est essentiellement le temps de la Restauration, avec le retour de la dynastie des Bourbons après l'Empire, quand la monarchie, dit-il en 1835, fut "attestée." Nous verrons son analyse des maux affectant la société mais aussi sa discussion sur les principes qui la régissent, en rapport surtout avec la position de la femme aristocrate.

La représentation et le fonctionnement des personnages dans les sphères du physique, du moral, et du social, sont le noyau du troisième chapitre, témoignant du grand génie de Balzac à donner à l'âme une dimension toute particulière.

D'invention réaliste et de vision divinatoire, la projection du chef d'œuvre balzacien est, par conséquent, paradoxal; et c'est essentiellement dans La Duchesse de Langeais, Les Secrets de la princesse de Cadignan, Le Cabinets des antiques, La Femme abandonnée, ainsi que Le Père Goriot, et Le Contrat de mariage, que nous pourrons l'apprécier. 
LA REPRESENTATION DE LA FEMME ARISTOCRATE EN PERIODE POST-REVOLUTIONNAIRE: BALZAC MORALISTE CHRETIEN ET APOLOGISTE DE LA PASSION

\author{
by \\ ISABELLE MARIE RENARD
}

A thesis submitted in partial fulfillment of the requirements for the degree of

\title{
MASTER OF ARTS \\ in \\ FRENCH
}

Portland State University

1996 


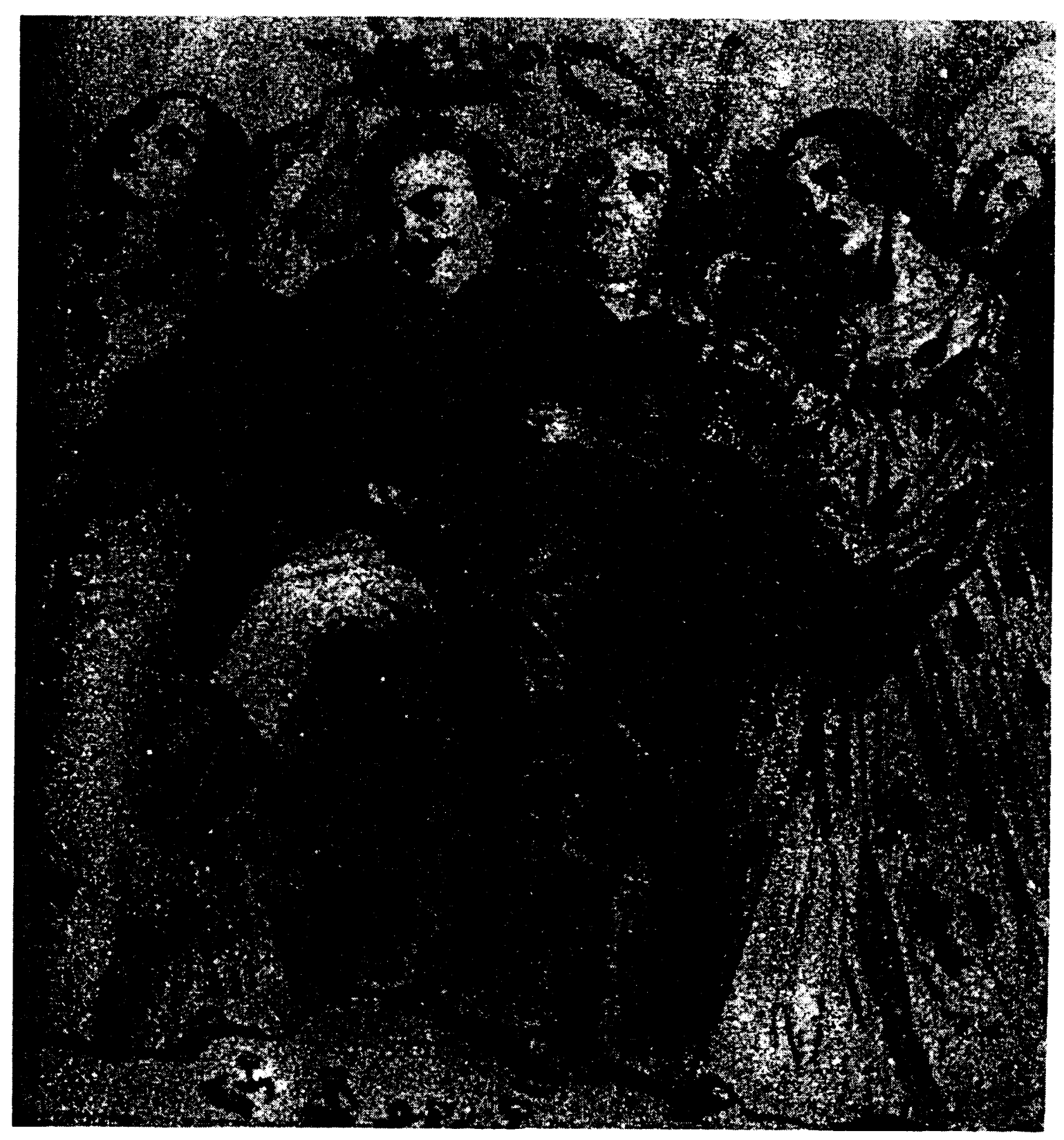




\section{INTRODUCTION}

Le but de mon étude est de considérer l'ambiguïté de la représentation de la femme du monde dans l'esthétique balzacienne. Pour ce faire, j'ai choisi trois êtres "supérieurs," selon Balzac: Mme de Beauséant, Mme de Langeais et Mme de Maufrigneuse, que nous verrons respectivement dans les œuvres suivantes: Le Père Goriot et Le Cabinet des antiques, Les Secrets de la princesse de Cadignan, et La Duchesse de Langeais. Le retour des mêmes personnages d'un roman à l'autre nous permet également de rencontrer d'autres femmes aristocrates nécessaires à notre analyse, dans un souci de comparaison: ce sont particulièrement (mais la liste n'est pas exhaustive), la marquise d'Espard, dans les lllusions perdues, et L'Interdiction, et Mme Evangelista et sa fille Nathalie dans Le Contrat de mariage.

Où se place l'ambiguïté? Nous verrons que d'un point de vue extérieur, ces coquettes se ressemblent étrangement mais qu'elles diffèrent dans leur personnalité plus intérieure. De ce fait diverses évolutions, diverses résolutions d'intrigues seront à l'étude. II apparaît clairement, tout au long de La Comédie humaine, que les femmes, occupant une grande partie de l'œuvre, sont l'objet d'une admiration certaine tout en étant l'objet d'une aversion parfois cruelle. C'est ce qui se retrouve dans le cas 
plus précis du déroulement des amours passionnées de cette minorité de femmes du monde, qui se voient constamment défendues mais finalement vouées à l'échec.

Pour un essai de résolution, je propose tout d'abord de considérer dans une première partie l'essentiel de la philosophie balzacienne. Celle-ci se trouve tout d'abord dans l'Avant-propos à La Comédie humaine, ainsi que dans les nombreux écrits constituant l'essentiel de la critique balzacienne. II réside en Balzac deux êtres, deux états conflictuels qui se retrouvent continuellement tout au long de l'œuvre: Balzac était défenseur de la morale chrétienne, mais sachant aussi reconnaître les instincts de tout être humain, il se fait aussi apologiste de la passion.

Ainsi il nous faudra étudier l'entendement de la vie et de I'humanité selon Balzac, qui s'appuie essentiellement sur une suprématie de l'âme. C'est aussi d'une comparaison entre l'Animalité et I'Humanité qu'il faut entendre le raisonnement balzacien.

Balzac se pose en défenseur de la Société, comme ayant une influence bienfaitrice sur la Nature. Nature et Société ont produit pour lui l'Etat Social qui sera alors à l'étude tout au long de son œuvre; ce sont les raisons du mouvement de la Société qui intriguèrent plus exactement le romancier et c'est aux sentiments qu'il accorde la place première, véritables moteurs de la vie. 
D'autre part il est nécessaire de comprendre pourquoi, selon le romancier, la morale est la plus importante des considérations sur l'homme, et le Christianisme, le plus grand élément d'Ordre Social. La religion enseigne à I'homme sa morale individuelle et, en moraliste chrétien, Balzac admire les êtres capables de dominer leurs passions, et il croit à la vertu. Mais l'homme est une créature faible, une créature d'instincts et d'émotions. Quelle en est l'influence sur l'amour et la passion? Nous verrons que l'amour est "une religion," un culte. Entre l'amour et la passion subsistent également des différences sur lesquelles Balzac nous éclairent, principalement dans son œuvre La Duchesse de Langeais. La passion est toute l'Humanité, et comment, dans l'absolu, se définit et évolue l'amour chez la femme?

Après avoir tenté de répondre à ces questions, et pour mieux considérer cette image de la femme, une des plus complexes de la condition humaine, il m'a semblé important de réserver une seconde partie au temps historique. En effet, dans la mesure où l'action de la plupart de ses nouvelles et romans se situe pendant la Restauration ou la Monarchie de Juillet, la peinture que Balzac fait de l'aristocratie y tient une place prépondérante. Nous tâcherons de comprendre la peinture de la situation politique mais aussi sociale, et conséquemment morale, de cette caste. L'aristocratie se place premièrement chez Balzac à Paris, mais nous verrons également que, nos héroïnes étudiées en faisant partie, l'aristocratie provinciale 
incarne l'envers des qualités du moyen-âge. La représentation de la femme fait écho aux préjugés et limitations imposées à cette époque. L'avidité de la société pour l'or et les possessions matérielles sont alors au premier rang et présentent des dangers certains pour l'évolution de la société dans son ensemble, mais surtout pour la noblesse, qui connaîtra alors sa lente agonie. Mais, plus spécifiquement, La Comédie humaine met en relief l'attitude de la société envers les femmes et le traitement des femmes. Où se trouvaient-elles sur l'échelle sociale? Comment les structures sociales du temps servaient-elles d'outils de répression des femmes?

Ainsi nous verrons que si le thème de l'adultère prédomine dans l'œuvre entière, il doit être entendu dans un contexte historique qui n'ouvrait aux femmes autre "profession" que celle d'être mariées, et ceci surtout pour les femmes aisées. Les vertus qui leur sont conférées devront être déplacées sur les bases d'une morale différente de celle que l'on pourrait défendre aujourd'hui au XXème siècle. L'évolution des personnages doit être prise en compte parallèlement à la transformation des mœurs que subit l'aristocratie. Leur sort s'inscrit dans I'histoire.

C'est dans une troisième partie que les qualités physiques et morales de ces créatures extraordinaires et extra-ordinaires seront analysées plus en détail, dans le texte. L'écriture balzacienne dévoilant tous les secrets du corps, il sera alors démontré comment le costume, ainsi que quelques parties du 
corps expriment non seulement des sentiments mais aussi la preuve de l'existence d'êtres "bien vivants." Ces femmes, dont le portrait extérieur n'offre qu'un "modèle" de mondaines égoïstes, incapables d'amour vrai, et qui se plaisent souvent à vivre loin de leur mari, peuvent aussi révéler des êtres "sujet," capables d'amour vrai. Nous verrons alors dans quelles circonstances cet amour vrai peut exister pour en arriver à leur résolution dans un destin tragique, ce qui sera l'objet de la quatrième partie. Traitées comme des "reines" par l'auteur, qui leur reconnaît un droit à la passion, pourquoi se trouvent-elles ainsi condamnées? II me semble que la seule chose que Balzac ne condamne véritablement soit la faiblesse. La tragédie de $\underline{\mathrm{L} a}$ Comédie humaine est impartiale aux êtres faibles. Et c'est dans une politique d'idéalisation et de vérité soutenue par l'auteur que se trouve précisément leur condamnation. 


\section{CHAPITRE I}

Conception balzacienne de l'homme et de la vie selon $\underline{L} a$ Comédie humaine

De l'importance de l'Etat Social, de la morale chrétienne, des passions et des sentiments d'amour

"La description des mœurs, l'analyse des caractères, la représentation d'une société fictive qui fait concurrence à l'état civil, le jugement porté sur le comportement d'un groupe défini par le milieu et l'histoire ne se comprennent qu'en fonction d'une vue supérieure qui découvre la nature et le destin de l'être humain."1

Et là fut le grand mérite de Balzac que l'on a, maintes fois et à juste titre, qualifié de visionnaire. Henri Gauthier disait encore que "I'homme extérieur" que surprend le faisceau de son regard, c'est "l'homme intérieur" que sa seconde vue pénètre. Mais avant de considérer l'examen balzacien de l'âme, révélée essentiellement par l'expression du corps, voyons les bases de son intellection sur l'existence humaine, nécessaire à notre projet d'étude de la représentation de la femme, et plus exactement de ce que Balzac appelait "la femme aristocratique."

L'entendement de la vie, selon Balzac, se fonde sur la différence originelle qui existe entre l'Animalité et I'Humanité,

1 Gauthier, Henri. L'Image de l'homme intérieur chez Balzac. Genève: Librairie DROZ, 1984. 
cohabitant en tout être. De cette idée naîtra ainsi La Comédie humaine, "d'une comparaison entre l'Humanité et l'Animalité."2 De même que, dans la nature, les espèces animales se différencient selon leur milieu, de même les hommes dans la société. Balzac divise, en d'autres termes l'humanité en espèces sociales analogues aux espèces zoologiques. Là se trouve le noyau de la théorie balzacienne que l'auteur avait résumée dans ces seize pages d'Avant-propos rédigées avec le calme et le recul nécessaire pour que la sagesse pratique trouve son chemin, seize pages qui lui demandèrent, avoue-t-il dans une lettre à Madame Hanska, plus de travail que la rédaction d'un roman entier. La nature humaine offrant une infinie variété à l'artiste, il n'a besoin que d'observer car "le hasard est le plus grand romancier du monde; pour être fécond, il n'y a qu'à l'étudier" (Avant-Propos, 13).

A cet état naturel s'ajoute, donc, l'influence non moins importante, voire même primordiale dans l'alchimie du comportement humain, de la Société. C'est ce que Balzac appelle l'Etat Social, qui a "des hasards que ne se permet pas la Nature, car il est la Nature plus la Société."3 Et "les raisons ou la raison de ces effets sociaux," c'est-à-dire les raisons du mouvement de la Société et de ses individus, devaient être

2 Balzac, Honoré de. Avant-Propos de La Comédie humaine. Pléiade, éd. Pierre-Georges Castex, I, p.11. 
mises à la lumière de son siècle et des siècles à venir. Balzac s'était donné la tâche, en ses propres termes, de "surprendre le sens caché de cet immense assemblage de fissures, de passions, et d'évènements;" et c'est en méditant sur les principes naturels et en quoi les sociétés s'écartent ou se rapprochent de la règle éternelle, du vrai, du "beau," qu'il a fait l'histoire oubliée de tous les historiens, I'histoire des mœurs.

Où se place donc l'individu dans la société, dans la conception et intuition balzacienne? La Comédie humaine, nous dit Gaston de Zélicourt dans son Monde de La Comédie humaine, nous présente:

une Humanité profondément individualisée, où pas une situation, pas un personnage n'est identique ou semblable à un autre. Les situations sont des tourbillons nés dans un grand mouvement d'ensemble qui ne reproduit jamais deux fois le même résultat, les personnages sont impossibles à confondre, foncièrement différenciés qu'ils sont par le dosage ou le 'Nombre' de leurs facultés, et par les influences que leur situation individuelle - ou collective a excercées sur leurs facultés et sur leurs comportements [...] Chaque individu et chaque situation est relié à la masse de l'ensemble par les hérédités, les influences et les conséquences" (Avant-Propos à La Ch, 15) 
Ainsi en vient-il à comparer ce tout compact et cohérent aux masses d'air dans l'atmosphère ou aux masses d'eau dans un océan. "Ainsi, depuis le plus grand jusqu'au plus petit des mondes, et depuis le plus petit monde jusqu'à la plus petite portion des êtres qui le composaient, tout était individuel et néanmoins tout était un" ( $X, 583)$. Ceci nous aide à mieux comprendre la structure d'ensemble de l'œuvre qui raconte des histoires dans des romans isolés que Balzac a désignées comme des "scènes" d'une grande comédie d'ensemble.

Mais ce qu'il est important de souligner dans toute analyse balzacienne, et qui nous servira ensuite de base fondamentale à tout le reste de notre étude, c'est l'idéologie de l'auteur concevant une opposition entre le principe animal et le principe spirituel, certes, mais aussi entre l'être intérieur et l'être extérieur, de même qu'entre le principe masculin et le principe féminin. L'aspect extérieur des vies individuelles ou collectives n'est qu'une manifestation sensible des idées; et ces idées appartiennent à ce que Balzac appelle "le monde moral," monde autonome. La Comédie humaine fut, autant qu'un constat d'une société, l'examen minutieux des phénomènes occultes de la puissance interne qu'il lui plaît de nommer tantôt l'âme, tantôt la pensée. Aussi, le principe fondamental est qu'il n'y a pas deux âmes semblables, puisque chacune d'entre elles a des idées différentes, des sensations différentes. Cependant, s'est révélé, chez Balzac, une vision systématique des êtres et une propension à donner une valeur typique à ses compositions que 
I'on découvre essentiellement dans cette citation tirée du Cabinet des antiques: "... entièrement femmes; elles sont entièrement mères, entièrement dévouées, entièrement nulles ou ennuyeuses" (III, 404).

La nature, les facultés et la finalité de l'esprit furent pour lui l'objet d'une longue interrogation de vingt années. De maximes en maximes, Balzac s'est appliqué à vouloir connaître l'être "moral." II se préoccupe beaucoup de la pensée des hommes, de leurs mobiles, de leur volonté d'agir. Le plus souvent, ce sont les idées et non les plaisirs des sens qui dirigent les personnages de La Comédie humaine. L'examen des activités psychiques incline Balzac, par conséquent, à distinguer deux êtres dans l'homme, deux puissances, deux phénomènes d'actions et de réactions et le conduit à chercher la résolution de ces dualités. II existe, ainsi, dans chaque individu une dualité intrinsèque aux sentiments, penchants, et caractéristiques des hommes, des femmes et des choses. "L'homme, dit-il par exemple, n'est ni bon ni méchant, il naît avec des instincts et des aptitudes. La Société [...] le perfectionne, le rend meilleur; mais l'intérêt développe aussi ces penchants mauvais." L'homme moral est particulièrement complexe mais l'idée balzacienne ne fait que rejoindre le "il y a deux hommes en moi" de Saint Paul qui ne fait que rejoindre l'expérience universelle: distinguer plusieurs âmes dans l'homme a de tout temps résolu le problème. Mais s'il semble finalement bien aisé de concevoir deux âmes dans un seul être, 
il est d'autant plus difficile de comprendre les subtiles combinaisons du corporel, du mental et de l'innommable essence supérieure qui forment l'unité de l'homme.

C'est à partir de cette constatation que l'on peut mieux comprendre le jeu antinomique d'une philosophie chrétienne et d'une conception romantique. Balzac reproduit le double sens de la vie. Sa vérité première reste le christianisme, la deuxième étant la Monarchie: "J'écris à la lueur de deux vérités, dit-il: la Religion et la Monarchie."4 La morale chrétienne est, pour lui, la plus importante des considérations sur l'être humain. Pour Balzac, moraliste chrétien, I'homme est un être faible, assez intelligent, cependant, pour comprendre l'enseignement religieux et moral, assez libre malgré le milieu et les habitudes acquises pour choisir le bien et pour être tenu responsable de ses actions. Mais pour Balzac romantique, l'être humain est une créature d'instincts et d'émotions, destinée à répondre à l'appel du plaisir des sens ou des grandes passions, comme l'estomac répond à la stimulation de l'odorat, à l'heure des repas. Ainsi, Balzac admire les rares individus qui dominent leurs passions, qui agissent avec constance et bonté. II croyait à la vertu et prônait plus que tout le culte de la volonté. Balzac détestait "la morale relâchée" de son temps et prêchait contre une société abominable. Le christianisme était pour lui le seul système de répression des tendances dépravées de l'homme qui pouvait seul 
intervenir comme élément d'Ordre Social. Les actions blâmables, les fautes, les crimes, depuis les plus légers jusqu'aux plus graves, $y$ trouvent toujours leur punition humaine ou divine, éclatante ou secrète. Elles ne sont pas, de plus, la majorité, comme il a été souvent avancé. Balzac s'était défendu lui-même de ces calomnies en prouvant, chiffres à l'appui, qu'il y a dans La Comédie humaine, plus de personnages vertueux que de personnages répréhensibles.

II se trouve aussi que, dans le monde de Balzac, la vertu trouve une définition nouvelle que celle à laquelle tout lecteur contemporain pourrait s'attendre. Balzac affirme que la vertu ne doit pas simplement se définir par l'adhérence aux règles, tout comme il ne peut exister de réelles vertus sans tentation. Si l'on prend par exemple le thème de l'adultère, prédominant dans La Comédie humaine, l'intervention d'un amant exemplaire est nécessaire à la vie monotone de ces jeunes femmes - qui créent aussi le désordre dans la société, dans l'esprit de l'auteur - que l'on marie très tôt à des banquiers, politiciens ou autres monstres inintéressants de la sorte qui le plus souvent prennent des maîtresses pour lesquelles ils se ruineront. La légalité de l'amant ne nous étonne plus une fois considérée dans son contexte historique. La femme prétend toujours, selon Balzac, avoir droit à un amant. C'est chose naturelle pour elle:

Si le métier d'honnête femme n'était que périlleux, passe encore [...] disait une vieille dame à Balzac, mais il ennuie 
et je n'ai jamais rencontré de femme vertueuse qui ne pensât jouer en dupe. Aussi, avant même qu'aucun amant ne se présente, une femme en discute pour ainsi dire la légalité. Elle subit un combat que se livrent en elle les devoirs, les lois, la religion et les désirs secrets d'une nature qui ne reçoit de frein que celui qu'elle s'impose.

C'est le combat des forces humaines et surtout des forces morales, c'est-à-dire intérieures à l'individu, qui préoccupent le romancier. Dans la réalité balzacienne, ces mots trouvent toute leur signification sur un plan social ainsi que sur un plan moral, deux systèmes largement contradictoires et inconciliables. L'existence simultanée de l'individu sur ces deux plans entraînent par conséquent des conflits incessants entre les deux complexes éthiques, conflits que Balzac traite, dans ses romans, comme le terrain sur lequel se jouent la plupart des drames personnels chez l'homme en Société. Mais les deux plans ne sont pas seulement contradictoires; ils sont aussi complémentaires: le jeu social exige souvent de hautes qualités morales dans tous les sens de ce mot. L'exigence morale posée par le social semble être monnaie courante mais il faut se méfier: lorsque Balzac en parle, il ne pense certes pas au lieu commun rebattu depuis l'antiquité qui exige du chef une moralité personnelle impeccable, presqu'au contraire. II préconise chez le chef social un machiavélisme et un manque de scrupules poussés jusque vers l'immoralisme. Son idéal de 
meneur social n'a rien de moral au sens édifiant de ce mot. Le caractère moral de l'obligation qui pèse sur l'individu pris dans le jeu social se présente sous deux faces qui correspondent à deux sens, d'ailleurs à peine distincts, du mot "moral." C'est tout d'abord une question de "forces morales" ou de qualités de la constitution psychique: le jeu social exige de l'énergie, de la lucidité chez le personnage qui doit faire face aux événements sur le plan social. Tout dépend, socialement parlant, de la qualité de l'appareil de l'individu: "la possession du pouvoir, quelqu'immense qu'il put être, ne donnait pas à la science de s'en servir. [...] Le pouvoir nous laisse tels que nous sommes et ne grandit que les grands" (PCh., IX, 231). Le moral comprend tout ce que l'individu enveloppe d'un sentiment de solidarité; le social ne commence qu'à la frontière qui sépare le "dedans avec moi" du "dehors avec eux." Et c'est aussi, en parallèle, la dualité existante entre le sentiment et la réalité que l'on retrouvera principalement dans le personnage de Diane de Maufrigneuse.

L'argent, le pouvoir, la distinction sociale sont, au yeux de l'auteur, les puissances maîtresses qui dominent les hommes de son temps. Les personnages dirigés par d'autres mobiles que ceux-là seront victimes de la Société. De cette constatation, sans doute, Jean Forest dans son Aristocratie balzacienne en est-il venu à considérer La Comédie humaine comme une tragédie, remarquant que "I'homme est tout entier contenu" dans l'œuvre. "On y voit sa force et ses espoirs, ses conquêtes et son orgueil, mais aussi sa faiblesse et ses défaites." Mais il ne faut 
pas oublier que son œuvre était une œuvre de littérature et non une œuvre historique, malgré le fait que nombre de critiques ont souvent considéré Balzac comme historien plutôt que comme romancier; il faut, par conséquent, prendre en considération les mots de Balzac lui-même quant à ce qu'il a voulu rendre, évaluant son travail dans le genre romanesque. Selon lui, le genre romanesque a pour loi de tendre vers le "beau idéal;" le roman doit être "le monde meilleur." Et cet idéal reste dans l'esprit de l'auteur à la dimension divine, dimension supérieure. Cet "auguste mensonge" d'un "beau idéal" ne libère pas, néanmoins, d'être vrai dans les détails; et c'est précisément dans la peinture de la femme que Balzac développe l'explication de cette théorie de l'idéalisation et de la vérité. C'est dans ce penchant à spiritualiser le réel et à l'exhausser que l'être féminin se trouve être une incarnation de l'idéal rêvé. "L'une des gloires de la société, c'est d'avoir créé la femme là où la Nature a fait une femelle; d'avoir créé la perpétuité du désir là où la Nature n'a pensé qu'à la perpétuité de l'espèce; d'avoir enfin inventé l'amour, la plus belle religion humaine."5

L'individu balzacien n'est autre qu'un produit, essentiellement déterminé par sa constitution initiale, les influences et la nature des situations auxquelles sa place dans le mouvement général l'expose, et agissant librement dans les limites que la situation a posées. II est à propos important de

5 Zweig, Stephan. Balzac: le roman de sa vie, 136-7. 
souligner cette remarque incontestablement pertinente de Gaston de Zélicourt sur Balzac et la liberté: "Balzac ne se livre jamais -sauf erreur- à une discussion sur la liberté car il semble que le degré de liberté devient dans La Comédie humaine, le rayon du cercle que le personnage peut encore dominer." Et c'est un point sur lequel il nous faudra nous arrêter plus longuement lorsque nous traiterons du caractère de la femme du monde mise en face de la réalité de l'amour et de la Société. Car il est un fait certain que ces femmes agissent librement dans les limites imposées par la Société, puisque souvent, voire même toujours -sauf erreur- elles payent d'un prix cher leur choix de contredire cette même Société.

C'est dans une perspective d'ensemble certes, qu'il faut analyser tout phénomène mais surtout, car Balzac se faisant constamment sociologue et psychologue, dans une perspective individuelle, envisagée et vécue de l'intérieur. Et ceci s'en trouve d'autant plus vrai lorsqu'il s'agit de considérer l'amour et la passion.

Beaucoup de critiques avancent que Balzac partageait une grande intimité avec les femmes; il n'est donc pas surprenant d'apprendre dans la biographie de l'auteur écrite par Stephan Zweig que:

dans le cercle étroit, mais immuable, de ses amitiés, ce sont les femmes qui dominent. Les neufs dixièmes de ses lettres, plus peut-être même, leurs sont adressées. C'est 
seulement devant elles qu'il peut céder à l'irrésistible besoin d'épancher de temps en temps en des confessions le trop plein éternel de son cœur [...] Jamais une lettre intime ne s'adresse à un homme, pas même aux plus grands et plus célèbres de ses contemporains." (17)

Il est toujours délicat, voire aventureux, de vouloir appliquer quelque théorie de psychanalyse à la production d'un écrivain, mais lorsque sa vie a déjà fait l'objet de centaine d'études et que ses œuvres ont été décousues dans toutes leurs largeurs, l'aventure ne présente plus autant de risques. Balzac, ayant souffert dans sa vie affective, et ayant souffert particulièrement de n'avoir pas eu de mère aimante et tendre, il ressent ce besoin profond de se faire comprendre et surtout aimé par les femmes. Sa mère était "un monstre et une monstruosité tout ensemble," nous apprend Balzac dans un terrible aveu fait à madame de Hanska. II poursuit:

Elle me hait pour mille raisons. Elle me haïssait déjà avant ma naissance [...] C'est une blessure qui ne peut guérir. Nous avons cru qu'elle était folle et avons consulté un médecin qui est son ami depuis trente trois ans. Mais il nous a dit: "Mais non, elle n'est pas folle. Elle est seulement méchante..." Ma mère est la cause de tous mes malheurs. 
Cette nature, prodigue dans tous les domaines, compense dans la recherche constante d'une femme qui devra être une mère, une amante, une sœur, et un soutien pour son travail, comme le fut Mme de Berny. Et sans doute pour toutes ces raisons que l'on vient d'énumérer, Balzac a produit dans son $œ u v r e$ une peinture toujours précise et vraie des sentiments de l'amour.6

Le manque d'amour maternel dont souffrait Balzac s'est exacerbé avec sa grande déception envers Mme de Castries et puis, plus généralement, sous le coup de l'expérience et avec l'âge, le romancier est devenu, après une sentimentalité doucereuse qui affectait ses œuvres de jeunesse, amer et vrai. Les sentiments sont indispensables, "la vie n'est que ce que nous la font les sentiments nous dit-il dans La Duchesse de Langeais (161). Il apparaît que Balzac, toujours partagé entre ses deux tendances éthiques contradictoires, n'arrive pas à reconnaître les sentiments d'amour comme totalement légitimes. II place ce sentiment très haut, lui accordant une dimension cosmique, nécessaire à toute existence, mais tout comme dans la religion chrétienne, où le sacrifice, la dévotion

$6 \mathrm{Si}$ je me permets de m'appuyer sur ces faits biographiques pour essayer de comprendre la psychanalyse des personnages balzaciens, c'est parce qu'ils ne sont pas, je pense, pure fiction et que la littérature n'est pas une pure abstraction. Bien au contraire, elle repose sur l'expérience humaine. L'art ou la littérature sont des représentations humaines de types ou d'émotions. 
entière à Dieu est impérative, l'amour semble requérir, chez Balzac, une joie, un sacrifice total de l'être à ce culte: "L'amour, c'est une croyance comme celle de l'immaculée conception de la Sainte Vierge; cela vient ou cela ne vient pas" (Le Contrat de mariage, 188). Mais lorque la fatalité nous envoie l'amour, il faut le traiter comme une religion: "L'amour est une religion, et son culte doit coûter plus cher que celui de toutes les autres religions" (La Duchesse de Langeais, V, 227).

II est important de souligner le fait que Balzac est prêt à accepter comme parfaitement réelles et sérieuses les émotions et autres signes de vie spirituelle qui naissent chez un sujet au contact de phénomènes trompeurs et artificiels. Mais si l'amour est un culte, la passion semble alors, chez Balzac plus "abordable" à l'humanité, voire même plus proche de sa nature, indispensable à toute manifestation de la vie: "La passion est toute l'humanité, nous dit-il. Sans elle, la Religion, I'Histoire, le Roman, l'Art seraient inutiles." Les explications de l'auteur lui-même sur ce qu'il entendait de l'amour et de la passion s'imposent à travers ce passage magnifique de La Duchesse de Langeais:

L'amour et la passion sont deux différents états de l'âme que poêtes et gens du monde, philosophes et niais confondent continuellement. L'amour comporte une mutualité de sentiments, une certitude de jouissances que rien n'altère, et un trop constant échange de plaisirs, une 
trop complète adhérence entre les cœurs pour ne pas exclure la jalousie. La possession est alors un moyen et non un but; une infidélité fait souffrir, mais ne détache pas; l'âme n'est ni plus ni moins ardente et troublée, elle est incessamment heureuse; enfin le désir nous le teint d'une même couleur: la vie est bleue comme l'est un ciel pur. La passion est le pressentiment de son infini auquel aspirent toutes les âmes souffrantes. La passion est un espoir qui peut-être sera trompé. Passion signifie à la fois souffrance et transition; la passion cesse quand l'espérance est morte. Hommes et femmes peuvent sans se déshonorer, concevoir plusieurs passions. Il est si naturel de s'élancer vers le bonheur! Mais il n'est dans la vie qu'un seul amour. Toutes les discussions écrites ou verbales, faites sur les sentiments, peuvent donc être résumées par ces deux questions: Est-ce une passion? Estce l'amour? (La Duchesse de Langeais, V, 220)

Vécu de l'intérieur, l'amour au féminin chez Balzac doit se manifester dans toute sa fureur. Rien n'est plus contraire à la nature des femmes qu'un amour tranquille et parfait: loin de là, elles ne désirent que des émotions. C'est un sexe qui est capable de "regretter les émotions de la peur" tant une femme peut être "avide de sensations extrêmes." 
Pour une femme bien éprise qui n'avait pas vu son amant depuis deux mois, ce rapide moment ne dût-il pas ressembler à cette phase de nos rêves où, fugitivement, notre vue embrase une nature sans horizons? Aussi, les femmes ou les jeunes gens peuvent-ils imaginer l'avidité stupide et délirante qu'exprimèrent les yeux de la duchesse. Quant aux hommes, si pendant leur jeunesse, ils ont éprouvé dans le paroxisme de leur première passion, ces phénomènes de la puissance nerveuse, plus tard ils les oublient si complètement, qu'ils arrivent à nier ces luxuriantes extases, le seul nom possible de ces magnifiques intuitions. L'extase religieuse est la folie de la pensée dégagée de ses liens corporels; tandis que, dans l'extase amoureuse, se confondent, s'unissent et s'embrassent les forces de nos deux natures. Quand une femme est en proie aux tyrannies furieuses sous lesquelles ployait madame de Langeais, les résolutions définitives se succèdent si rapidement, qu'il est impossible d'en rendre compte. Les pensées naissent alors les unes des autres, et courent dans l'âme comme ces nuages emportés par le vent sur un fond grisâtre qui voile le soleil. (La Duchesse de Langeais, V, 226)

Mais il appartiendra à notre troisième chapitre sur la représentation de la femme d'analyser en détail les manifestations intérieures des sentiments ainsi que l'évolution 
de la destinée individuelle, tout autant que la manière dont se manifeste à l'extérieur leur vie intérieure, la façon dont chacune d'elles répond aux défis lancés par l'existence. Tout dépend de la vie de l'individu lui-même mais aussi de la vie du groupe auquel il appartient, dans des rapports plus ou moins indirects selon la place qu'occupe l'individu, le sort des grandes collectivités, de la Société et de l'Humanité dans son évolution historique. 


\section{CHAPITRE II}

\section{Interprétation balzacienne de l'histoire}

Pour mieux comprendre l'évolution des personnages étudiés, leurs états sociaux, il est important de les considérer dans leur cadre historique. II sera bon par exemple de rappeler quelques faits historiques et de les voir interpréter par l'auteur lui-même. Certes, ce sont les événements qui affectent l'aristocratie sur lesquels nous nous arrêterons; son action et ses réactions aux différentes étapes de l'histoire, tant bien à Paris qu'en province, et le rôle social des femmes de sa classe qui implique inévitablement sa gente masculine correspondante, elle-même moralement faible et abêtie, selon Balzac. L'importance des femmes doit être considérée dans le cadre des nuances subtiles de classe qui nous apparaissent à travers le texte balzacien. C'est particulièrement dans le contexte d'une ascension flagrante de l'importance de l'argent, et conséquemment de la bourgeoisie financière, qu'elle s'opère à Paris ou en Banlieue, qu'une lutte permanente, sous-jacente mais bouillonnante, entre la noblesse et cette même bourgeoisie entrave et modifie les lois des relations humaines, les lois des sentiments.

Balzac donne à son âge héroïque, tributaire en ceci du Moyen-Age, l'aspect d'une pyramide où successivement, à partir du sommet, s'échelonnent la royauté, l'aristocratie féodale, le 
clergé, la bourgeoisie et le peuple. Comme le signale Pierre Barbéris dans Le Monde de Balzac:

L'aristocratie a pris une place de plus en plus considérable au fur et à mesure que s'avançait l'œuvre. Balzac, pris par ses mirages et ses illusions, lui a donné une importance croissante. C'est la classe que Balzac, avec le plus de force, a voulu reconstituer.(167)

La transformation que subit l'aristocratie dans l'œuvre est parallèle à l'histoire: Balzac dépeint le pluralisme de l'aristocratie dans les œuvres qui se déroulent avant la fin de la Restauration, et en particulier jusqu'en 1824, date de la mort de Louis XVIII. Là, il s'efforce de décrire ses différentes composantes coexistant dans une alliance qui leur permet de garder leur identité. Dans des œuvres comme La duchesse de Langeais, Le Père Goriot, qui se situent respectivement en 1819 et 1824-29, les différents groupes maintiennent leur identité et coexistent dans une alliance dominée par la puissance conférée à la pairie créée par l'ordonnance du 20 août 1815.

La vieille aristocratie partage alors le pouvoir avec la noblesse d'Empire dans le cadre d'une monarchie restaurée mais transformée par les gouvernements de la première république et du premier empire. Le pluralisme politique que déploie l'aristocratie sous le règne de Louis XVIII décroît progressivement sous celui de Charles $X$. Le refus de Charles $X$ 
de poursuivre une politique de réconciliation privilégie temporairement la vieille aristocratie qui vit un triomphe provisoire. A partir de 1830 la transformation du pluralisme persiste, mais sous une forme nouvelle. Sous la Monarchie de Juillet la vieille aristocratie n'a plus qu'une apparence de grandeur et elle doit céder pratiquement tout son pouvoir à une nouvelle alliance formée, cette fois de la Noblesse d'Empire et de la Bourgeoisie financière. Balzac se voulait historien plus que romancier avait-il dit, et ses qualités d'observateur de l'être humain s'ajoutent à ses qualités de visionnaire. Ainsi, il met en scène, dans La Comédie humaine, chaque type de particule sociale que ce vaste ensemble peut renfermer.

La vieille aristocratie de La Comédie humaine, celle du Faubourg Saint-Germain, à laquelle apartiennent la vicomtesse de Beauséant, La Duchesse de Langeais, le Comte Grandlieu ou les Navarreins, ainsi que celle qui vit en province comme les d'Estrignon, les Rastignac ou les Vandenesse, retrouve son pouvoir et une partie de ses privilèges avec le retour des Bourbons. Dès le premier retour de Louis XVIII, les nobles des familles patriciennes reprennent leur ancienne place dans la société. Les officiers retrouvent leur grade et leur rôle dans l'armée. Les fils des anciennes familles aristocratiques reprennent également leurs fonctions diplomatiques, à l'instar du frère de Félix de Vandenesse, Charles, dans Le Lys dans la vallée quand il est "envoyé au congrès de Vienne" en 1814 (IX, 1099). Cette vieille aristocratie n'est pas politiquement unie 
et elle se divise entre ceux qui critiquent, ceux qui tolèrent ou ceux qui soutiennent la politique de réconciliation sociale de Louis XVIII.

Pendant la Restauration cette ancienne aristocratie cohabite avec l'aristocratie d'Empire illustrée par des personnages comme le colonel Chabert, qui est comte, le général Montriveau, qui lui est marquis, ou le baron de Nucingen. Dans La Comédie humaine cette cohabitation est symbolisée par la définition que Balzac donne du Faubourg Saint Germain au début de La Duchesse de Langeais:

Ce que I'on nomme en France le faubourg Saint-Germain n'est ni un quartier ni une secte, ni une institution, ni rien qui se puisse nettement exprimer. La Place Royale, le Faubourg Saint-Honoré, La Chaussée d'Antin possèdent également des hôtels où se respire l'air du faubourg SaintGermain. Ainsi tout le Faubourg n'est pas dans le Faubourg. (V, 923-4)

La Seine ne sépare plus totalement les deux mondes de l'aristocratie légitimiste qui habite le Faubourg Saint-Germain sur la rive gauche et la nouvelle aristocratie qui demeure dans le quartier dans la Chaussée D'Antin sur la rive droite. Le mélange social n'est pas limité à l'installation de certaines familles de l'ancienne aristocratie rive droite. Monsieur de 
Montriveau habite rue de Seine, près de la duchesse de Langeais, chez qui il se rend quotidiennement.

Au début du règne de Louis XVIII, les jeunes gens des meilleures familles patriciennes semblent promis à participer à la destinée de cette nouvelle monarchie côte à côte avec les vaillants soldats de Napoléon, comme le général Montriveau. Dans La Duchesse de Langeais, la transformation rapide de la situation de ce dernier dans l'armée illustre la politique de bascule de Louis XVIII qui, tout en voulant consolider les assises de l'ancienne aristocratie, ménage les soldats annoblis de Napoléon. "Après les adieux de Fontainebleau, Montriveau, quoique noble et titré fut mis en demi-solde" (V, 942). Montriveau avait obtenu le grade de colonel de la garde pendant les Cent-Jours mais ne fut pas reconnu par le gouvernement royal; partir pour une expédition en Egypte fera de lui un héros. Quand Madame de Langeais, fille du Duc de Navarreins, symbole le plus pur de la vieille aristocratie, décide de faire la conquête de ce soldat, "alors rétabli sur les cadres, dans son grade" et "admis dans La Garde Royale" (V, 943), il est devenu la coqueluche du Faubourg Saint-Germain. En 1818,

le gouvernement royal, qui cherchait à s'attacher les hommes de mérite afin de donner de la force à l'armée, fit alors quelques concessions aux anciens officiers dont la 
loyauté et le caractère connu offrait des garanties de fidélité. (V, 934)

L'intérêt de Mme de Langeais pour le général Montriveau, "un homme de Bonaparte" (V, 1014), illustre la fusion que Louis XVIII préconisait; cet intérêt est alors aiguisé car le général de Montriveau représente "le type le plus complet de la nature à la fois supérieure et faible, grande et petite, de sa caste" (V, 934). Les parents de la duchesse sont d'ailleurs prêts à accepter le général comme un des leurs, comme le montre le commentaire de la princesse de Blamont-Chauvry pendant la réunion qu'ils tiennent après "l'incartade de leur jeune parente" (V, 1012). Monsieur le duc de Navarreins, le vidame de Pamiers, la vieille princesse de Blamont-Chauvry, le duc de Chauvry et le duc Grandlieu sont réunis pour empêcher la jeune femme de détruire complètement sa réputation après le coup d'éclat qu'elle a fait en laissant sa voiture stationner devant la demeure du général pour le convaincre de son amour. Quand la tante de la duchesse affirme que "les Montriveau sont anciens et fort bien alliés, ils tiennent à toute la haute noblesse de Bourgogne" (V, 1014), elle donne bénédiction à l'amour des deux jeunes gens, qui concrétise l'union des deux noblesses. Malheureusement le nombre des nobles éclairés qui ont compris que le pouvoir nouveau de l'aristocratie devait s'adapter à une période nouvelle est limité. L'ensemble de la caste représentée par le Faubourg SaintGermain, fidèle à ses traditions, n'est pas prêt de reconnaître 
que "si les temps sont changés," "les armes" aussi (V, 928). La noblesse française n'a pas pu se régénérer de la même façon que l'aristocratie anglaise, ce que déplore Balzac dans le long portrait qu'il fait de l'entourage de Mme de Langeais.'

Dans son article "La Duchesse de Langeais et le romanesque balzacien," Arlette Michel qualifie le faubourg Saint-Germain de "monde fossilisé" qui est "rebelle au mouvement et incapable de penser le temps en termes de renouvellement," et aussi de "société bloquée" (94). Dans L'Aristocratie balzacienne, Jean Forest illustre aussi la fossilisation de cette caste en considérant les préoccupations superficielles des deux piliers de La Comédie humaine:

MM. de Navarreins et de Grandlieu, qui figurent au nombre des personnages les plus importants de La Restauration dominent la cour comme le Faubourg. Ils laissent pour leur part apparaître dans leur conversation l'insignifiance de leurs préoccupations, axées sur d'éternelles questions de généalogie, d'alliance et de fortunes. (215)

D'autre part Balzac regrette que cette aristocratie se soit laissée corrompre par l'argent sans savoir utiliser le pouvoir pour dominer:

1 La Duchesse de Langeais, $V$, 923-34 
Au lieu de se montrer protecteur comme un Grand, le faubourg Saint-Germain fut avide comme un parvenu. Du jour où il fut prouvé à la nation la plus intellligente du monde que la noblesse restaurée organisait le pouvoir et le budget à son profit, ce jour, elle fut mortellement malade. $(\mathrm{V}, 930)$

L'importance que prend l'argent dans la vie des vieilles familles nobles est un thème central dans La Comédie humaine. Dans Ĺ'Interdiction la perspicacité du juge Popinot lui permet de deviner que ce sont les dettes de la marquise d'Espard qui la poussent à vouloir empêcher son mari de restituer à la famille Jeanrenaud une partie de sa fortune acquise grâce à la confiscation des biens des protestants sous le règne de Louis XIV.

La Restauration est un grand affrontement entre libéraux et royalistes. Les esprits en 1815 interdisaient la réapparition des privilèges que la Révolution avait balayés: Napoléon avait consolidé les avantages obtenus par la classe populaire, et hissé la classe moyenne au premier degré de sa hiérarchie; l'une et l'autre refusaient désormais la compartimentation qui caractérisait l'ancien régime, et que d'aucuns ne souhaitaient remettre à I'honneur. Balzac connaissait bien le grand dénuement de l'aristocratie, installée par les alliés dans un siècle dont elle ignorait les lois consécutives: "L'art, la science et l'argent bornent le triangle social où s'inscrit l'écu du 
pouvoir, et d'où doit procéder la moderne aristocratie."2 L'ancienne ne brillait dans aucun de ces domaines, équivalents modernes des empires de la vieille Europe, et quelque puissance fût-elle, celle de l'Europe coalisée, elle ne pouvait la renverser. "En 1814, mais surtout en 1820, la noblesse française avait à dominer l'époque la plus instruite, la bourgeoisie la plus aristocratique, le pays le plus femelle du monde."2

L'aristocratie n'est pas consciente d'avoir été victime de la politique de bascule du souverain qui tout en voulant consolider les assises de la vieille aristocratie et en limitant ses ambitions n'a pas su contrôler l'ascension politique de la bourgeoisie. Dans La Duchesse de Langeais, Balzac utilise souvent le terme "éphémère" pour qualifier la vie de l'aristocratie du faubourg Saint Germain, et il annonce ainsi la révolution de 1830 qui confirme son déclin. Le pouvoir de la vieille aristocratie n'est plus qu'une illusion. C'est maintenant la noblesse d'Empire alliée à la bourgeoisie financière qui contrôle le gouvernement ainsi que l'expansion économique et sociale. Pierre Barbéris confirme que "l'aristocratie qu'a vue Balzac est une aristocratie arrivée au dernier terme de sa décadence" (216).

Dans les œuvres où l'action a lieu avant 1830, et en particulier avant 1824, le pluralisme de l'aristocratie

2 La Duchesse de Langeais, $V 147$

${ }^{3}$ Le Cabinet des antiques, IV, 459-60 
représente l'espoir déçu du légitimiste, tandis que dans les œuvres où l'action se passe sous la Monarchie de Juillet, la dissolution du pluralisme de l'aristocratie reflète totalement, de la part du partisan des Bourbons, le rejet de la nouvelle monarchie de Louis-Philippe corrompue par le matérialisme bourgeois. C'est dans les provinces que se ressent certainement le plus cette suprématie de l'argent et des préoccupations matérielles. Et c'est dans l'autorité de Diane de Maufrigneuse face à M. d'Estrignon que ce fait apparaît clairement:

Vous êtes donc fous, ici? reprit la duchesse. Mes chers enfants, il n'y a plus de noblesse, il n'y a plus que de l'aristocratie. (...) Vous serez bien plus nobles que vous ne l'êtes quand vous aurez de l'argent."3

Dans cet univers plat où ne subsistaient que des préoccupations matérielles, Paris faisait cruellement ressentir son absence. Au contact de Madame de Beauséant, parisienne en exil, Gaston de Nueil ressuscite: "Il foulait un vrai tapis de Paris, revoyait le type distingué, les formes frêles de la parisienne, sa grâce exquise, et sa négligence des effets cherchés qui nuisent tant aux femmes de province." 4 Les créatures exceptionnelles qui peuplent les salons parisiens

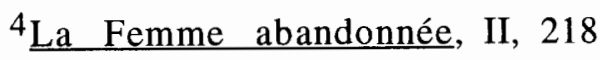


sont, pour Balzac, dotées d'une âme exceptionnelle qui résiste à l'influence délétère de la province.

Toute ville de province, pour peu qu'elle abrite quelques noms antiques, possède son cénacle, véritable refuge d'âmes affolées. Le nouveau gouvernement fleurit un peu partout, minuscule à Provins, puissant, quoique déclassé aux yeux du siècle dans la riche et bourgeoise ville de Bordeaux où "écrasé par les hautes et mouvantes fortunes des villes maritimes, ce faubourg Saint-Germain (...) répondait par son dédain au faste qu'étalait alors le commerce, les administrations et les affaires." 5 Le plus célèbre de ces salons est naturellement le cabinet des antiques, où dominent plus nettement que partout ailleurs la contemplation inintelligente des gloires du passé et la peur stérilisante du monde moderne. Ces hauts lieux de la noblesse provinciale, malgré leur ridicule, et peut-être parce qu'ils n'étaient sensibles qu'aux étrangers, étaient la convoîtise d'opulents bourgeois qui n'aspiraient plus qu'à la satisfaction d'y être admis. Comme leur requête rencontrait inévitablement une résistance farouche, ces calmes refuges d'âmes blessées se transformèrent souvent en champs de bataille. Le refus d'y recevoir même les représentants de la petite noblesse, jugée indigne d'un si grand honneur, trace la ligne de démarcation qui sépare les éléments conservateurs des éléments progressistes, les petits nobles, vexés, se rangeant assez tôt du côté de la

5 Le Contrat de mariage, III, 83 
bourgeoisie. La noblesse se scinde en deux partis ennemis. Du Bousquier sera du nombre des refusés:

les plans ambitieux qu'il avait formés d'abord, manquèrent une première fois par le refus de Mlle d'Estrignon, de qui l'alliance lui aurait donné l'entrée dans le faubourg Saint-Germain de la province $[\ldots]^{6}$

La province, en résistant avec succès aux influences parisiennes, Balzac la voit abêtir chaque année un peu plus ceux de ses fils qui lui seront restés fidèles, de gré ou de force, après les orages éphémères de leur jeunesse. Le comte de Mannerville se souvient-il du temps de ses folies? C'est pour mieux leur tourner le dos et imposer à son fils la sagesse chevrotante d'un vieillard devenu "progressivement économe, avare et ladre."7 Et pourtant cinquante ans plus tôt cette momie trouvait l'énergie et le courage, après avoir dissipé la fortune de son père, de la reconstituer avec succès. Avec quelle éloquence Balzac ne parle-t-il pas de la maladie sénile des provinciaux incurables:

Le vieux gentilhomme le menait [son fils Paul] chaque soir dans une vieille voiture, traînée par de vieux chevaux mal attelés, accompagné de vieux laquais mal habillés, dans

\footnotetext{
6 Le Cabinet des antiques, II, 339.

7 Le Contrat de mariage , III, 82.
} 
une société royaliste, composée des débris de la noblesse parlementaire et de la noblesse d'épée. ${ }^{8}$

D'autre part, la famille Watteville représente bien le bête immobilisme de la province qu'à voulu rendre le romancier. Le Baron n'aspire qu'à la tranquillité et pour arriver à ses fins s'incline servilement devant les volontés de la baronne. La Société qu'elle préside se soumet également à son autorité: en politique, elle est toute l'aristocratie de Besançon. Et toute l'aristocratie de Besançon tient tout entière entre les murs de son salon, d'où l'intelligence a fui, vraisemblablement pour lui faire place.

S'il existe, donc, dans l'esprit de Balzac, un gouffre de différences très net entre la société du Faubourg-SaintGermain et celle des villes de province, ce sont les femmes qui l'illustrent peut-être encore mieux. Les provinciales tiennent leurs yeux braqués sur Paris, obsédées par la mode qui s'y crée, et "croient être affublées d'un châle et d'un bonnet." 9 Elles font tous les ans l'achat de deux chapeaux, dont elles prélèvent le prix, qui les angoisse fort, sur leur budget personnel, font ensuite assaut de vertu dans les salons quand elles ne sont pas occupées à prendre des airs de reine dans un cabriolet d'osier qu'elles prennent pour un carosse. Car, selon Balzac, leur

\footnotetext{
8 Ibid, 83.

9 La Femme abandonnée, II, 208.
} 
dignité ne souffre en rien de leurs médiocres moyens. Tous sont dans la gêne et les moins fortunés connaissent la misère.

La noblesse provinciale, sauf de rares exceptions, a perdu confiance en elle et se réfugie dans la contemplation stérile d'un passé qu'elle recrée. Sa grande faiblesse économique ne rend pas seule compte de cet abandon, la dégénéréscence de la race y jouant un rôle prédominant. Là-dessus Balzac est formel et les pièces à conviction de ce procès qu'il mène tambour battant sont innombrables. L'aristocratie terrienne, moralement et physiquement diminuée, cachée dans ses vieux châteaux ou ses antiques hôtels, ne peut plus remonter la pente abrupte de sa déchéance. Son inertie l'en empêche, d'abord. Ses chefs de famille n'ont de volonté que vélléitaire et, comme Monsieur de l'Estorade fils, remettent leur destin entre les mains de leurs épouses. M. de Morsauf, et l'on ne peut imputer sa faiblesse aux circonstances de son émigration, dont de plus fortes volontés seraient sorties trempées, ne comprend ni sa femme, ni sa situation, ni son époque et ne doit le succès de son entreprise agricole qu'aux bons conseils de sa femme, qui dirige tout à son insu. Car même si l'institution du Code Napoléon en 1804 avait rendu les femmes impuissantes dans la structure sociale où elles se trouvaient assujetties à un véritable régime militaire, il semble que la réalité balzacienne leur ait donné plus de pouvoir que l'histoire voulait bien leur en accorder. Le code soulignait, par exemple, l'infériorité inhérente des femmes. Selon l'article 213 du Code Civil les maris avaient une 
emprise absolue sur leurs épouses. Les femmes étaient censées obéïr à leurs maris à tout moment. Elles n'avaient pas le droit de protester. En tant qu'êtres "inférieurs," elles devaient accepter leur servitude. En fait, elles étaient obligées de rendre hommage à leurs maris pour cette "protection paternelle." L'article 1124 du Code a mis les femmes sur le même plan que les enfants mineurs et les fous. De cette façon, nous dit Brenta, dans son article intitulé "Corps infirmes, corps infâmes," le Code n'aura fait que de continuer "la tâche de l'abjection totale de la femme commencée bien avant la révolution elle-même." Elle continue, fait sur lequel je reste en désaccord et pour lequel je tiens à ouvrir une parenthèse, "Balzac a malheureusement soutenu cette attitude misogyne dans son œuvre." II est vrai que certains passages de sa Physiologie du mariage ainsi que les premières Scènes de la vie privée, par exemple, sont anti-féministes; mais en apparence. Les sages conseils maritaux prodigués aux époux en vue d'assurer leur domination absolue dans le cercle de la famille, sont donnés sur un fond d'ironie et de scandale; tous les chapitres sérieux y tendent vers le but opposé, qui est d'alléger le fardeau moral et social que la société charge sur les femmes. N'oublions pas que Balzac ne semble pas concevoir la destinée féminine heureuse et harmonieuse dans le cadre du mariage. 10

10 C'est dans le dernier chapitre de ma démonstration que ce problème de la représentation de la femme sera plus développé; la femme en tant que 
Les femmes de la noblesse avaient toujours rempli dans l'obscurité de leur foyer les tâches primordiales qui leur étaient assignées. Maître Mathias, ahuri par les nouvelles mœurs, rappelle à Paul de Mannerville combien celles-ci rompent avec les anciennes:

Vous me direz que ceci est une vieille méthode de nos ancêtres; mais, dans les familles nobles, M. le comte, une femme légitime doit faire les enfants et les bien élever: comme le disait la duchesse de Sully, la femme du grand Sully, une femme n'est pas un instrument de plaisir, mais I'honneur et la vertu de la maison. 11

Mais, elles s'étaient muées, dans l'œuvre, en grandes dames et appartenaient au monde. Le vide qui s'était créé de ce fait au sein des foyers permettaient l'intime transformation des générations appelées à affronter les orages de la fin du siècle. La femme noble, considérée son éducation, n'était guère préparée à affronter la tourmente. Elle était, disait-on, apte tout au plus à recevoir dans les salons, les seuls champs de batailles de l'aristocratie. Les nobles y font assaut de la vanité que le siècle avait substitué à l'orgueil ancien. Mme Evangélista, recommandant à sa fille d'être "la grande dame qui

sujet, pouvant atteindre les plus hautes sphères de la Pensée, et détenant un pouvoir véritable.

11 Le Contrat de mariage, III, 178 
représente le luxe et le plaisir de la maison [...],"12 loin de céder au goût du jour, propose un idéal que seules quelques vieilles dames réalisent encore, la Grande Dame étant morte avec "l'entourage grandiose" du siècle dernier. Ce siècle, étant donné la démission des hommes, et son caractère galant, s'il est en politique celui de Louis $X V$, est pour l'aristocratie celui de la femme noble, qui exerce une royauté mondaine incontestasble. Une coquetterie franche qui s'affiche sans honte, finesse de grâce et d'esprit, sachant supérieurement causer, tout ceci s'exprime dans la princesse de Blamont-Chauvry. Elle porte dans sa vieillesse comblée de tous les égards dûs à son rang, à son savoir et à son âge, le charme de la jeunesse. L'esprit de la cour n'a pas quitté "le plus petit débris du règne de Louis XV."13

Sous la Restauration, les femmes, autrefois épouses et mères, ne permettent plus aux hommes, qu'elles combattent, de compter sur leur appui moral. Créatures de la société, elles vivent de ces luttes et rivalisent avec tous ceux qui prétendent les dominer, sans distinction de sexes. Le caractère de cette femme, celui aussi de l'existence à laquelle elle était vouée, n'échappe ni au bourgeois qu'est Horace Bianchon, ni aux aristocrates que sont Rastignac et Ronquerolles. Bianchon voit en elle une créature dépourvue d'âme, de tendresse, et même de sentiment maternel, ce en quoi la marquise d'Espard lui donne raison. II les a vues hors de leur domaine, les salons, et a pu

\section{Ibid, 164}

13 La Duchesse de Langeais, V, 228 
constater qu'elles s'affichent, dans leur intimité, tous les vices qu'elles avait soigneusement cachés en société, la maladie rendant alors son dévoilement inévitable. La femme du monde, pervertie, dénaturée, condamne la Restauration. 14

Ronquerolles, qui n'a plus depuis longtemps la naïveté de Montriveau, voit en elle une créature désincarnée: "elle ne sent que par la tête, elle a un cœur dans la tête. [...] Elle ne peut plaire et de fait elle plaît énormément, qu'aux hommes ambitieux qui ont 'tué le mandarin."'15

Rastignac, n'obéissant qu'à des impératifs mondains, apprécie en elles un moyen de parvenir parfaitement adapté à ses besoins, un outil tranchant que l'usage n'ébrèche pas, le produit le plus typique de la Restauration. Aussi donne-t-il volontier raison à Bianchon:

Mais tu n'es pas dans la question. [...] Epousez un ange! II faut s'enterrer dans son bonheur au fond d'une campagne. [...] Ta femme aimante ne mène à rien, une femme du monde mène à tout, elle est le diamant avec lequel un homme coupe toutes les vitres, quand il n'a pas la clef d'or avec laquelle toutes les portes s'ouvrent. 16

\footnotetext{
14 L'Interdiction, III, 13

15 La Duchesse de Langeais, III 220

16 L'Interdiction, III ,15
} 
L'amour est lié à l'ancienne noblesse, au contact régénateur du sol ancestral, à la Vie. L'ambition pure, la désincarnation, l'égoïste besoin de briller en société est lié à l'exercice d'une fausse virilité qui s'exhibe, justement, de préférence dans la compagnie de femmes dénuées de cœur. Le Faubourg est une impasse pour l'aristocratie, qui y perd le goût du sol et celui de se fondre dans la masse des Français: seuls les honneurs, les insignes du pouvoir l'intéressent à Paris. II n'est pas étonnant dans ces conditions, que la noblesse de province ait été négligée gravement par le gouvernement:17 il tournait le dos à la sagesse politique en refusant de reconnaître qu'en elle résidait son unique chance de survie, qui passait par la reconnaissance de la primauté de la masse. Celle-ci abandonnée, avait plusieurs raisons de croire à l'inutilité de l'aristocratie.

D'une importance capitale est le fait que l'aristocratie, celle de Paris surtout, ne croyait plus à elle-même, et qu'elle affichait ostensiblement le cynisme qui avait remplacé l'ancienne foi en une mission sacrée. Les provinciaux toujours convaincus de leur qualité, quoique privée des moyens de l'inscrire dans la réalité, soit économique soit politique, découvrent en arrivant à Paris que les liens du sang ne jouent plus en leur faveur. II s'y est créée une caste à part, le Faubourg, qui méconnaît aussi bien les qualités anciennes de la 
noblesse provinciale que les vertus nouvelles de la bourgeoisie. De la première il retint la morgue, de la seconde, il adopte le goût de l'argent et méprise avec une inconscience égale nobles ruinés et bourgeois parvenus.

La faiblesse morale des jeunes nobles, ultime conséquence de leur dégénéréscence, annulait ce que leur personnalité pouvait compter de promesses. Le plus grand se reconnaît davantage en Paul de Mannerville, "un de ces hommes [...] qui tiennent beaucoup de la femme,"18 qu'en la mâle figure du Général Montriveau. Le plus illustre d'entre eux, plus encore que Lucien de Rubempré, en qui des origines mi-nobles mibourgeoises se confondent pour composer le type d'une faiblesse plus universelle, est assurément Victurnien d'Estrignon, "ce voluptueux mauvais sujet, indigne de son nom."19 Incapable de se fixer comme de résister aux tentations, tombant dans tous les pièges, il aime par dessus tout la sensation d'être emporté par le courant. II sera rejeté par Diane de Maufrigneuse, lassée de "cette âme [...] frappée d'une épouvantable faiblesse à son centre."20

\footnotetext{
18 Le Contrat de mariage, III 92

19 Le Cabinet des Antiques, IV 402

20 Ibid, 375
} 


\section{CHAPITRE III}

\section{Representation de la femme aristocrate}

II s'agit de considérer maintenant l'importance du rôle de la femme dans l'œuvre de Balzac, et plus précisément celui des femmes aristocratiques. Face à la faiblesse morale des jeunes nobles dont nous venons de parler les femmes du monde prennent le pouvoir; revêtant leur masque et costumes d'ange, elles se parent d'une virilité féminine qui leur donne un pouvoir de domination certain, ainsi qu'une très grande liberté d'action. D'autres comme la duchesse de Langeais en particulier, en minorité dans l'œuvre, conservent une vérité extérieure semblable, et paraissent faire usage de cette liberté à mauvais escient: elles seront alors condamnées par Balzac pour avoir eu la faiblesse d'avoir provoqué et d'avoir ressenti le sentiment vrai de l'amour. Mais n'est-il pas une étrange condamnation de la part d'un homme qui se voulait défenseur de la passion amoureuse!

Les contemporains de Balzac ont souvent parlé de son succès auprès du beau sexe. On pense à la gravure contemporaine de son temps dans laquelle Granville montre les célébrités littéraires participant à une "Grande Course au Clocher Académie," et où I'on voit Balzac soutenu par des femmes. Gautier affirme qu' "il y a dans son œuvre comme une odeur de femme: odor di femina; quand on y entre, on entend 
derrière les portes qui se referment sur les marches de l'escalier dérobé des frou-frou de soie et des craquements de bottines."1 Déclarant lui aussi que le grand succès de Balzac est venu des lectrices, Gozlan a porté un témoignage analogue:

Elles ont adoré en lui l'homme qui a su avec éloquence, par de l'ingénuosité encore plus que par la vérité, prolonger indéfiniment chez elles l'âge d'aimer et surtout celui d'être aimées. Cette galanterie en quarante ou cinquante volumes, les a exaltées comme le ferait le fanatisme d'une religion nouvelle."2

En 1834 déjà La Revue des Deux Mondes publiait un article où Sainte Beuve affirmait que c'est précisément en flattant certaines fibres du cœur féminin que Balzac a su gagner la moitié du public qui est si essentielle aux succès de librairie. Le critique explique cette conquête par une théorie selon laquelle le romancier a su se présenter aux femmes comme "un confesseur un peu médecin"qui savait leurs "secrets sensibles et sensuels." 3

On a vu dans le premier chapitre que Balzac a tout de même une certaine tendance à systématiser, même s'il a été

${ }^{1}$ Honoré de Balzac, 159.

2 Balzac en pantoufles, 18.

${ }^{3}$ Article recueilli dans Les Grands Ecrivains français par Sainte Beuve, Garnier, 1927, I, 140-1. 
reconnu par certains que le stéréotype n'existait pas chez lui, . Dans le portrait qu'il fait de la femme à la mode (classe à $^{4}$ laquelle appartiennent les personnages étudiés dans cet essai), sous la Restauration et Louis Philippe, on y voit, comme chacun pourrait s'y attendre, un excès d'attention sur les frivolités et choses superficielles:

Elle ne pensait qu'à ses succès, à sa toilette; elle allait aux Bouffons, à l'opéra, au bal; se levait tard, se promenait au Bois, dînait en ville ou donnait elle-même à dîner. [...] Là est la raison de l'insensibilité, du froid des salons. Les belles âmes restent dans la solitude, les natures faibles et tendres succombent, il ne reste que des galets qui maintiennent l'océan social dans ses bornes en se faisant frotter, arrondir par le flot, sans s'user. 5

Voilà sans doute une des raisons pour lesquelles certaines femmes du monde ne périssent pas à ce métier (cf. Natalie dans Le Contrat); sachant mener leurs organisations avec une main de fer et par conséquent avec peu de cœur et des estomacs solides, elles vainquent sur les autres qui se laissent prendre aux griffes d'un "homme de fer." C'est ce que l'innocent Paul de Mannerville n'a certainement pas su être:

4 Terme reconnu pour désigner ce que l'époque avait aussi appeller la coquette. Cf. Les Mondains, 303.

5 Le Contrat de mariage, 391. 
Ta femme résistait admirablement à cette vie, elle y semblait habituée, elle apparaisait toujours fraîche et belle; pour moi, la conclusion était facile à tirer; elle ne t'aimait pas, et tu l'aimait comme un fou. Pour faire jaillir l'amour dans cette nature silicieuse, il fallait un homme de fer. 6

Le charme féminin s'exprime très souvent par la nervosité, la recherche minutieuse d'élégance, la coquetterie, le caprice, et la violence. Très souvent Balzac fait usage de la métaphore féminine; de cette façon il peut souligner les traits caractéristiques qu'il reconnaissait dans la femme. Le retour constant à cette métaphore féminine nous rend compte, une fois de plus, de la place occupée par les femmes dans le monde de Balzac, mais c'est aussi, selon moi, une façon d'adoucir les quelques petites choses désagréables qu'il avait à communiquer à ses chères lectrices, puisqu'il les mettaient à I'honneur en même temps. La France et la femme étant ainsi juxtaposées, non seulement enrichit-il son écriture, mais les mots semblent un peu moins cruels, car atteignant leur cible principale, les femmes, indirectement:

6 Ibid, 396. 
[...] La France, femme capricieuse, veut être heureuse ou battue à son gré. [...] Jamais nation ne fut plus complaisante, elle était alors comme une femme fatiguée qui devient facile; jamais pouvoir ne fit alors plus de maladresse: la France et la femme aiment mieux les fautes. ${ }^{7}$

De cette façon, les défauts, la jalousie, l'exigence, l'amour de force, ainsi que diverses formes de vénalité sont stéréotypés, . Mais, chez Balzac, la métaphore féminine revêt généralement une valeur positive. Dans l'ensemble elle évoque des qualités morales ou des visions de beauté; c'est par exemple l'image de Paris qui revient dans nombre de ses œuvres à laquelle nous pensons instantanément. Une des plus belles métaphores de la femme se trouve, par exemple, en toute dernière page du Père Goriot. Là, se dessine l'image de Paris comme une femme, aux formes courbes, allongée: "Rastignac, resté seul, fit quelques pas vers le haut du cimetière et vit Paris tortueusement couché le long des deux rives de la Seine, où commencaient à briller les lumières." 8 . Les images spiritualistes s'ajoutent un trait physiologique dominant sur le plan de l'instinct. Le corps de la femme est source de mille voluptés, les courbes et rondeurs de son corps la rendent excessivement sensuelle à tel point que la métaphore féminine

${ }^{7}$ La Duchesse de Langeais, V, 150.

8 Le Père Goriot, II, 1085. 
fait voir toute passion comme un déplacement d'énergie sexuelle.

Les femmes aristocratiques, possédant de par leur naissance, la richesse, l'éducation et l'esprit, "le goût supérieur," détiennent ainsi les meilleures armes qui soient pour combattre les hommes, et surtout ceux qui veulent les dominer. Mais pour cela il faut bien évidemment être dépourvue de cœur, être incapable de sentiments amoureux. L'activité des femmes malfaisantes est intense dans La Comédie humaine. Est-ce sans doute le malheureux père Goriot qui en est la victime la plus connue. Mais ce cas n'est pas rare. Homme trop confiant, le colonel Chabert a commis l'erreur d'épouser une prostituée du Palais Royal; en guise de remerciement sa femme trouve le moyen de l'envoyer finir ses jours à Bicêtre. Dans L'Interdiction, la Marquise d'Espard ne se montre pas plus humaine avec un mari gênant. Voulant être seule maîtresse d'une fortune dont elle a besoin pour payer ses dettes, cette femme rusée et perfide a recours à des calomnies qui ont pour but de faire croire à la folie d'un homme qui a le malheur de ne pas être assez malhonnête, car il a donné tout son bien pour liquider une dette d'honneur. D'un point de vue social, le thème de la domination féminine trouve toute sa justification en accord avec l'interprétation de l'histoire que Balzac présente dans la Comédie humaine. Dans La Duchesse de Langeais, la Princesse de Blamont-Chauvry accuse les hommes de n'être qu'une génération de petits poitrinaires à lunettes d'écailles, et 
affirme que le jour est venu où les femmes doivent remplacer ceux-ci devenus incapables d'énergie:

Aujourd'hui, vraiment, il semblerait que les rôles soient changés, et que les femmes doivent se dévouer pour les hommes. Ces messieurs valent moins et s'estiment davantage. Croyez ma chère, toutes les aventures qui sont devenues publiques et dont on s'arme aujourd'hui pour assassiner notre bon Louis XV, étaient d'abord secrètes.

Sans un tas de poétriaux, de rimailleurs, de moralistes qui entretenaient nos femmes de chambre et en écrivaient les calomnies, notre époque aurait eu littérairement des mœurs. [...]

En effet ce qui naît de cette protestation féminine est une tendance vers un renversement des rôles; ainsi pouvons-nous parler de virilité féminine dans le monde balzacien.

C'est dans Le Contrat de Mariage que le romancier nous donne un des exemples les plus frappants d'une entreprise de domination féminine. Madame Evangélista a su marier sa fille Natalie avec Paul de Mannerville, brave jeune homme qui ne sait pas profiter des conseils que lui donne de Marsay et qui commet l'erreur capitale d'aimer aveuglément. Aussi ne comprend-il pas que sa belle-mère l'utilise comme un instrument. Si cette dame a décidé d'employer ses talents au profit de son gendre c'est "afin de goûter sous son nom les plaisirs de la puissance." 
Et Balzac ajoute que beaucoup d'hommes sont ainsi "les paravents d'ambition féminine inconnues." Le notaire Solonnet explique en outre qu'en France la femme heureuse est celle qui est maîtresse au logis, opinion que partage Madame Evangélista. Cette personne redoutable déclare que les femmes doivent réapprendre à gouverner leurs maris, art qui aurait connu un certain déclin grâce à telle "manie de la famille" répandu par Jean-Jacques. Dans les discussions domestiques, déclare Madame Evangélista dans le Contrat de Mariage,"les femmes ont un dernier argument, qui consiste à dire "je ne veux pas."9 C'est alors que "la force féminine se montre toute entière," ajoute-telle.

Nombreuses aussi sont les héroïnes de Balzac qui nous permettent de parler de sentiments romantiques et d'innovations psychologiques; mais notables sont celles qui appartiennent à la "classe supérieure." II n'y a que trois femmes de cette sorte dans tout l'opus, mais leur présence n'est qu'omniprésente, "for they are the model for lesser social queens and stand at the apex along the glittering ladder of champions over the human heart."10 Femmes à la mode, ces femmes extraordinaires ne doivent pas être confondues avec les nombreuses insignifiantes coquettes, et arrivistes qui les imitent. Elles sont elles-mêmes sans merci dans leur jalousie, compétition, mais ne perdent jamais leur goût supérieur et leur

${ }^{9}$ Le Contrat de mariage, III, 166.

10 Mc Cormick, Diana. Sailing on the Balzacian Ocean of Women, 129. 
dignité. Ainsi Mme de Beauséant, Mme de Langeais et Mme de Maufrigneuse forment un trio remarquable.

Le personnage de Mme de Bauséant est sans doute l'un des plus touchants. Après avoir perdu l'homme qu'elle aimait, Adjudo Pinto, à une simple Mlle de Rochefide, riche et eligible pour le mariage, elle se retire seule, dans la solitude la plus totale dans un petit village de Normandie. Ces évènements sont relatés dans quelques chapitres du Père Goriot.

Mme de Langeais est plus qu'une comparse dans Le Père Goriot, c'est une langue bien aiguisée, un diamant des salons parisiens. Cependant, dans le volume intitulée La Duchesse de Langeais la prude, élégante et coquette se transforme en un personnage tragique qui, blessée au plus profond par son amour, se retire dans un couvent pour y mourir.

Le destin de Diane de Maufrigneuse, princesse de Cadignan, n'est point tragique mais ironiquement triomphant. Elle aussi a régné dans les salons parisiens, mais alors qu'elle approche les quarantes ans, elle désire ardemment trouver le véritable amour de sa vie. C'est une reine qui vieillit et qui plutôt que d'essayer de reconquérir sa couronne se bat pour ses droits en tant que femme. Elle joue alors toutes ses cartes, prête à gagner ou tout perdre, afin de conquérir le grand écrivain d'Arthez, qui dans son innocence la croit toute innocente aussi. Au lieu de le garder loin de ceux qui pourraient lui ouvrir les yeux, elle l'envoie à une soirée où elle sait que des amis envieux se feront un plaisir de l'instruire sur ses nombreux amants qu'elles avait eus dans 
le passé. "Elle ne savait quel parti prendre au cas où d'Arthez croirait le monde qui disait vrai, au lieu de la croire, elle qui mentait."11 || y a bien sûr une certaine ironie dans son triomphe: le bonheur du couple échappe à la catégorie des amours bourgeois et au banal contentement mais ne repose au contraire que sur un mensonge qui se doit d'être constamment renouvellé: "Elle était condamnée à le tromper, car elle voulait rester pour lui l'actrice sublime qui avait joué la comédie à ses yeux." C'est alors un mensonge qui prend forme artistique, devant toujours trouver un nouveau visage.

Ces femmes gardent tous les éléments nécessaires de l'idéalisation recherchée par Balzac. Jean-Pierre Richard, dans son chapitre sur la dramatisation et l'idéalisation de l'être féminin, nous a montré que dans le Paris décrit ou inventé par Balzac, "la richesse, la beauté, la naissance et l'esprit donnent à la femme qui les possède une royauté non moins prestigieuse que celle que l'on conférait avec de l'huile sainte."12

Nouvelle incarnation d'une certaine aristocratie, la Duchesse de Langeais jouit elle aussi d'une espèce de royauté officieuse. En elle aussi se résume les défauts et la grandeur d'une caste que Balzac critique et admire à la fois. Comme il convient à une souveraine, Antoinette de Langeais ne paraît jamais dans le monde sans sa cour de femme distinguée: "Reine de la mode, elle avait ses dames d'atours, qui reproduisaient

11 Les Secrets de la princesse de Cadignan, VI, 64.

12 Etudes sur le romantisme, 196. 
ailleurs ses manières et son esprit."13 Elle sait "dominer" la société où elle vit, et se plaît à recueillir les hommages, tout en restant maîtresse d'elle-même. Quintessence d'une certaine féminité balzacienne, elle fait figure d'être privilégié dont la splendeur et la puissance sont remarquables.

Telle qu'elle paraît dans Le Cabinet des antiques et dans Les Secrets de la princesse de Cadigan, épisodes marquants de son existence avant et après la révolution de 1830, Diane de Maufrigneuse donne encore plus d'attraits à la figure de la femme aristocratique, maîtresse de la société, amante idéale. Diane est "une des dix plus jolies femmes de Paris, avouées, reconnues." Adorée d'une foule de courtisans, elle est "une des reines de Paris, une reine éclatante."14 Et ses titres à cet honneur sont les mêmes que ceux de Foedora et d'Antoinette: la naissance, la beauté et l'esprit.

La supériorité certaines dont jouissent ces femmes se traduit par une grande liberté d'action: méprisant les entraves qui lient les femmes ordinaires, Diane est prête à braver le monde, et ne craint pas de s'attacher ouvertement à Victurnien d'Esgrignon. Quand celui-ci est en prison, elle montre l'étendue de son dévouement et de son énergie: croyant qu'il partage sa fermeté d'âme, elle veut donner à son amant le poison qui le sauverait du déshonneur. C'est la même force intérieure qui la soutient lors de la chute de sa fortune en 1830; refusant

${ }^{13}$ La Duchesse de Langeais, V, 211.

14 Les Secrets de la princesse de Cadignan, VI, 15. 
d'abdiquer et d'accepter l'inactivité, la trépignante duchesse vise à la prééminence dans un nouveau domaine, celui de la politique. Aussi n'hésite-t-elle pas à tremper dans l'insurrection vendéenne, réunissant chez elle des conspirateurs, et cela en même temps qu'elle reçoit le premier ministre du nouveau régime. Ce dévouement courageux aux intérêts de sa classe lui vaut l'admiration de l'auteur, qui montre en elle le type même de la femme aristocratique et qui la décrit comme un être à part:

Ce fut chez la princesse que le maréchal auquel nous devons la conquête de l'Afrique, eut, lors de la tentative de MADAME en Vendée, ... tant était grande l'obscurité de la princesse, tant sa détresse excitait peu la défiance du gouvernement actuel. [...] II y eut de la noblesse et de la grandeur à risquer ainsi le fils unique de l'héritier d'une maison historique [...] il n'y eut chez la princesse de Cadignan aucun calcul. 15

Les femmes comme Diane peuvent arriver à ce que la sensibilité a de plus élevée, de même qu'elles peuvent soumettre leurs sentiments à une domination absolue. Une Diane de Maufrigneuse Cadignan possède cette faculté qu'avait Napoléon de tout subordonner à son intelligence et à sa volonté. 
Maîtresse d'elle-même, elle l'est aussi de son univers. Autre fait important: ce personnage constitue un démenti d'un certain féminisme tapageur. C'est Diane elle-même qui le précise:

Vous avez fini, vous autres écrivains [déclare-t-elle à d'Arthez] par rendre bien ridicule les femmes qui se prétendent méconnues, qui sont mal mariées, qui se font dramatiques, intéressantes, ce qui me semble du dernier bourgeois. On plie et tout est dit, ou l'on résiste et l'on s'amuse[...] Quelle sottise aux femmes de se plaindre! Si elles n'ont pas été les plus fortes, elles ont manqué d'esprit, de tact, de finessse, elles méritent leur sort. Ne sont-elles pas reines en France?16

Diane a bien réalisé ce programme de force et de liberté. En "don Juan femelle, "elle a passé sa vie à s'amuser. Loin d'elle cet "aveu d'impuissance" des femmes qui quittent leur mari. Dernier d'une assez longue liste d'amants, d'Arthez défend les actions de sa maîtresse: pourquoi le beau sexe ne prendrait-il pas de temps en temps "une revanche," demande-t-il? Et pour bien prendre cette revanche, les femmes sont naturellement poussées à jouer un rôle; car toute femme ambitieuse et intelligente de La Comédie humaine revêt un masque afin de réussir sur la "scène" parisienne. Martha Moss remarque

16 Ibid, VI, 43. 
d'ailleurs très justement que Balzac aura choisi d'intituler son œuvre "comédie," métaphore théâtrale qui se révêle d'autant plus dans le vocabulaire utilisé tout au long de l'œuvre. Dans ce monde de la noblesse le combat des femmes reste identique à celui des femmes du peuple, mais cela reste d'autant plus marqué que les mariages de convenance étaient de règle dans "le monde;" c'est la lutte éternelle des femmes contre un système patriarcal qui ne leur accorde aucun droit à s'affirmer en tant que "sujet."

Pour s'en défendre, Madame Evangelista est "la meilleure femme du monde en apparence,"17 "but a master of Machiavelian intrigue in reality. She hides her true nature from her weak son-in-law, Paul de Mannerville, and sets out to rob him of his fortune by the signing of a marriage contract which she prepares with her lawyer."18 Les éléments de la métaphore du théâtre se trouvent ainsi clairement dans le vocabulaire utilisé par l'auteur. Les personnages deviennent les comédiens d'un grand jeu, le jeu de la vie: "La scène était préparée entre eux comme les joueurs préparent les cartes pour une partie où l'on ruinera le pigeon."19 La princesse de Cadignan pour sa part excelle dans la pratique d'un accent digne de la plus habile comédienne.

17 Le Contrat de mariage, II, 386.

${ }^{18}$ Moss, Martha. "The Masks of Men and Women in Balzac's Comédie humaine", The French Review V-50: 3 Feb 1977: 446-53.

19 Le Contrat de mariage, II, 379. 
Voyons ainsi de quelle manière Balzac s'est appliqué à dépeindre, avec une impression de vrai extraordinaire, ces femmes aristocratiques qui aiment à se déguiser pour mieux manipuler une société où les valeurs se placent plus certainement dans l'apparence extérieure que dans le substantiel; ou bien dans le cas de nos trois héroïnes, comment ces femmes sentimentales deviennent alors actrices pour mieux sauver leur honneur ou I'honneur de celui qu'elles aiment. C'est, en premier lieu, grâce au costume que le processus créateur de Balzac peut se déployer, ce processus "qui consiste à 'empeindre' et la pensée de la nature et la réalité dans la pensée; autrement dit, à faire passer une vision intérieure dans le réel en lui donnant une forme matérielle et à transformer le réel observé, vécu, en idéal artistique."16 Dans la peinture d'une vérité extérieure, le costume occupe, par conséquent, une place considérable. Balzac savait bien la signification profonde du costume, ce qu'il appelle "l'esprit du chiffon."17 II s'attache aussi une valeur historique, sociale, psychologique ou morale au genre d'étoffes dont sont faits les costumes et les toilettes, à leurs couleurs, à leur odeur, à leur son, à la façon de les porter aussi bien qu'à leur opposition et à leur évolution. La liberté est laissée aux personnages de varier tel ou tel détail de leur costume pour exprimer tel sentiment ou telle idée. C'est ce que

16 G ancil, Farag. Le Costume et le caractère dans La Comédie humaine de Balzac. Le Caire: Les Livres de France, 1974. p.5.

17 Traité de la vie élégante, Librairie Nouvelle, 1854, 82. 
le romancier appelle le "Code Femelle: "cette "collection de petites lois" qui permettent aux femmes de "se moquer des lois que les hommes ont faites."18 Les femmes de l'aristocratie, elles, s'habillaient en se souvenant d'avantage du passé, et justement Balzac déplore la disparition du "grand siècle" avec ses atours de soie.

Le costume et la toilette, la façon de les porter dévoilent les différents caractères, les types d'hommes et de femmes les plus simples et les plus complexes avec leur milieu, leurs fonctions, leurs habitudes, leurs manies et leurs passions, leur monstruosité ou leur poésie, leur bonheur ou leur drame. Un rien dans les vêtements peint leurs réactions psychologiques les plus profondes, les plus délicates ou les mieux cachées:

Tout en écoutant les aventures du général Armand de Montriveau, la duchesse de Langeais jouait avec son écharpe, la tordait, la déchirait par des mouvements d'impatience qui semblaient accuser le mécontement et de profondes réflexions. Elle acheva de détruire son écharpe, comme un enfant qui, jouant avec une fleur, finit par arracher tous les pétales; elle la roula, la jeta loin d'elle et put ainsi montrer son cou de cygne. ${ }^{19}$

18 Splendeurs et misères des courtisanes, V 998.

19 La Duchesse de Langeais, V, 176-7. 
Aussi les tremblements de voile de la carmélite peuvent trahir la vive émotion qu'elle éprouva. ${ }^{20}$

Le costume contribue essentiellement, chez Balzac, à révéler la personnalité des personnages; les simples plis d'une robe lui suffisent, par exemple, à traduire la noblesse d'une femme et les couleurs portent une valeur significative. Farag Gancil s'est appliqué dans son ouvrage à étudier ces correspondances. II aura ainsi constaté que le blanc représente l'innocence, la distinction et la grandeur; le rose, l'amour idéal pur et platonique tel que celui d'Henriette de Morsauf dans Le Lys dans la vallée; le vert, l'espérance et les grandes aventures; le bleu, l'élégance, la richesse raffinée, le goût, le rêve, la vertu fière et digne:

Elle [Mme de Maufrigneuse] avait mis une robe de velour bleu à grandes manches traînantes, à corsage apparent une de ces guimpe en tulle légerment froncée, et bordée de bleu, montant à quatre doigts de son cou, et couvrant les épaules, comme on en voit dans quelques portraits de Raphaël."21

Le pourpre sera porteur de spiritualité; la couleur brune, de pauvreté et d'ascétisme, comme par exemple "la longue robe brune" de Mlle Armande d'Estrignon qui a voulu se sacrifier et

21 Les Secrets de la princesse de cadignan, VI, 32. 
demeurer vieille fille pour se charger de l'éducation du jeune comte d'Estrignon. Le noir renferme toute une gamme d'indications; il n'exprime pas seulement le deuil mais encore le sacrifice et le dévouement des maîtresses pour leurs amants, la distinction des hommes politiques, des grands artistes, celle des gens braves et des vieillards et la monstruosité des criminels. Le rouge et les couleurs éclatantes sont très souvent dans le costume du peuple. Quoiqu'il en soit, tous les amoureux de La Comédie humaine savent bien l'importance vitale de la toilette et de la parure.22 lls savent bien que l'équation de l'amour comprend aussi bien l'élégance et le goût que le cœur; l'amour fait déployer des flôts de soie et de cachemire, "des jupes savamment étalées qui couvrent tout et tout en lumière."23 Les toilettes sont étudiées et les amoureuses se mettent "sur le pied de guerre"24 et les éléments de la toilette deviennent des "munitions," des "armes" de séduction indispensables au renforcement de la tactique galante et à la victoire finale. La princesse de Cadignan se sera préparée habilement pour aller dîner chez son amie, la marquise d'Espard, où elle compte bien séduire l'artiste. Et quand à l'âge de quarante ans, "voyant venir la terrible faillite de l'amour, [..]" un unique élément de parure ne suffit plus: "sa femme de chambre l'avait coiffée de quelques bruyères blanches habilement posées

22 La Duchesse de Langeais, V, 224.

23 Les Secrets de La princesse de Cadignan. VI, 30.

24 Le Père Goriot, II, 864. 
dans ses cascades de cheveux blonds auxquelles elle devait sa célébrité." 25

Mais le costume peut aussi bien n'être que tromperie, comme celui de Diane de Maufrigneuse; comme le visage et les manières il exprime une nature d'emprunt. Elle porte une "robe blanche," une "ceinture de petite fille, un "fatras de virginités en mousseline." 25 Des plumes d'ange sont collées sur un être qui n'est pas un ange. Blancheur, finesse de la taille, transparence vaporeuse des voiles, voilà qui suffit à estomper les lourdeurs charnelles et à suggérer l'immatérialité. En chaque toilette Diane semble évoluer à travers un nuage: dans "un de ses négligés coquets [...] qui lui permettaient de commencer son rôle d'ange dès onze heures du matin;"26 en robe de nuit quand Chesnel l'implorant de restituer la somme remise par Victurnien, "elle s'élança vers son secrétaire en passant devant le notaire comme un de ces anges qui traversent les vignettes de Lamartine. 9 On ne s'étonne plus que Diane paraisse "un ange pour tout le monde," en tout lieu, à toute heure, et même dans sa chambre à coucher. Tous ces costumes sont de séduisantes trahisons, des mirages ensorcellants. Celui de Diane est fausse parure et loin de signifier sa nature authentique.

25 Les Secrets de la Princesse de Cadignan, VI, 29.

26 Ibid, 31. 
"Sous les manières d'une Agnès Romantique27 se cache l'âme d'une Célimène, "Célimène qui représente la femme aristocratique."28 D'un côté des poses d'ingénue, de l'autre le froid calcul d'une femme du grand monde, sa splendeur et sa dureté. "Elle était délicieuse à voir en colombe, étouffant la lueur de ses regards sous les franges dorées de ses cils."29 Ecoutant d'Estrignon lui offrir la fuite, pour elle le salut, mais pour lui le scandale et la ruine,

elle tenait ses yeux attachés aux yeux du comte; mais des myriades de sentiments passaient sous leur bleu, comme des lueurs d'orages entre deux nuées. Elle avait le front calme, la bouche sérieuse d'attention, sérieuse d'amour, les lèvres nouées à celles de Victurnien [...] _ Bien, dit la duchesse qui, au lieu d'être livrée à l'amour qu'elle exprimait, était livrée à de profondes combinaisons qu'elle gardait pour elle. 30

Aussi, ce type de femme faisant l'ange pour séduire se retrouve dans le portrait de la marquise d'Espard qui "agitait ses grandes manches comme si ç'eut été des ailes."31 "Malheur à

27 Ibid, 39

28 Ibid, 56

29 Ibid, 60

30 Ibid, 46

31 Le Cabinet des Antiques, IV, 384. 
celui qui devait "s'enfoncer" dans son amour et "payer le compte de ses ailes," compte qui atteignait la somme de "cent mille francs."32 La marquise d'Espard est un monstre de fausseté dans L'Interdiction où voulant réchauffer sa nature froide par des émotions, des plaisirs et des jouissances, cette femme est "un sexe dans le cerveau."33 "Jamais ni la gaze, ni la soie, ni la mousseline, n'ont été plus habilement entortillées autour d'un mensonge!"34 Les mouvements onduleux de sa longue robe trahissait les secrets mouvements du dangereux reptile.

Dans ses œuvres, Balzac ne se contente pas d'analyser les costumes de ses personnages mais évidemment, leurs amours, leurs ambitions, leurs grandeurs ou leurs crimes, mais aussi l'état de leurs nerfs, leurs névroses, leurs humeurs, les sécrétions de leurs glandes, la mesure de leur angle facial.

Il émane, effectivement, de ces personnages balzaciens un don de vie "extra-ordinaire," une puissance par laquelle ils se trouvent généreusement emportés. Pour expliquer selon quelles formes il s'active, il faut se référer à ces quelques phrases importantes de la Recherche de l'absolu:

toute vie implique une combustion [...] toutes les fois que la Nature a perfectionné un appareil, que dans un but

32 Ibid, 391

33 L'Interdiction, III, 14.

34 Ibid, 13 
ignoré, elle y a jeté le sentiment, l'instinct, ou l'intelligence, ces trois organismes veulent une combustion dont l'activité est en raison directe du résultat obtenu. 35

Toute existence se manifeste donc comme incendie. Le corps se place sous le signe de la flamme. Et dans les termes de Jean-Pierre Richard, tout personnage balzacien se trouve ainsi mû par "un même brasier passionnel."

Pour appuyer cette reconnaissance d'une énergie intérieure volcanique, c'est à l'étude faite par Françoise Frangi dans son article parû dans L'Année balzacienne de 1971 que je tiens à faire référence maintenant. Son Essai de lecture stylistique sur La Duchesse de Langeais souligne essentiellement la présence d'un registre des images de la flamme et de la lumière. Françoise Frangi distingue ainsi trois états: celui du choc, celui de la flamme, et celui de la lumière qui représenteraient les trois phases d'un cycle, celui de la vie. La perspective balzacienne se poserait dans l'optique d'une vie qui commencerait avec un choc, grandirait avec la flamme et s'épanouirait dans la lumière. Avant le choc se trouverait un état sans vie alors qu'après la lumière se révèlent des images de rayonnement, de soleil. Ces images de soleil et de

35 La Recherche de 1'absolu, IX, 537 
rayonnement correspondent alors à la notion de fluide vital, qui figure sous la désignation de "souffle divin:"

Pour Balzac, nous dit-elle, Dieu, ou plus exactement la Divinité, c'est le principe qui permet la vie [...] Tout être vivant, selon le degré de pénétration de ce fluide en lui, se trouve placé à chaque instant dans une phase déterminée du cycle de la vie.(238)

Cette projection du fluide se produira ainsi dans l'écriture à travers les couleurs. Les couleurs sont d'une importance primordiale dans l'œuvre de Balzac et tout spécialement lorsque la vie se trouve pour un moment bouleversée ou même défiée. Les couleurs sont les repères qui marquent le passage ou l'appartenance à tel ou tel état de la vie.

Ainsi, l'auteur méditant sur l'amour relève que "L'âme n'est ni plus ni moins ardente ou troublée, elle est incessament heureuse; le désir, étendu par un souffle divin, d'un bout à l'autre sur l'immensité du temps nous le teint d'une même couleur; la vie est bleue comme l'est un ciel pur" (242). Tandis que le blanc sera l'indice de la fausse lumière des salons, le bleu est l'indice de la vraie lumière. Le système de cycle de la vie tel que Françoise Frangi s'applique à le définir est "une structure de l'écriture balzacienne: il est en effet le mode fondamental de perception et de transcription de la réalité par le romancier" (246). 
II existe d'autre part un code du comportement spontané du corps. Dans l'emportement de la passion surtout il s'exprime indéniablement. Le corps désiré ou désirant se trouve porteur d'une sorte d'écriture immédiate et c'est en termes d'inflammation, de pulsion, de caractère d'explosivité que l'on définira toute grande passion chez Balzac. Ainsi l'amour de Montriveau est "volcanique éruption."36 Aussi, tel mot de la Duchesse de Langeais provoque chez Montriveau des "secousses électriques." L'être se déclare alors de la façon la plus littérale comme un feu d'artifice. Et pour se libérer de cette brutale flagrance sexuelle, il suffira que le corps amoureux s'éloigne, s'envole, s'évapore dans un lointain d'espace ou de mémoire. Ce type d'expression aéro-lumineuse prend donc aussi une valeur de sublimation. Mais le déploiement de l'être se saisit du corps tout entier, et en particulier des cheveux du front, des lèvres, et des pores.

Chez les personnages féminins la chevelure prendra une valeur d'affirmation certes moins brutale, mais elle continuera à afficher (sur un mode sourdement sexuel) sa liaison avec l'humeur profonde. De cette façon "les cheveux fins et cendrés d'Henriette de Morsauf "la faisaient souvent souffrir," et ces souffrances, imagine Balzac, "étaient sans doute causées par de subites réactions du sang vers la tête."37

${ }^{36}$ La Duchesse de Langeais, V, 131

37 Le Lys dans la vallée, VIII, 797 
Le front, lui, est un thème de clôture; mais d'une clôture qui se chargerait surtout de nous faire pressentir, et comme apercevoir en elle l'assaut même des forces qu'elle aurait pour fonction de contenir. Derrière sa façade, plus ou moins ample et dégagée, s'imaginera toujours la pression quasi physique des pensées. L'espace frontal ne sert qu'à enveloppper et annoncer le volume du mental.

Transpirer est aussi excréter de façon spontanée le trop plein humoral. Tandis qu'"elle sentait poindre cet amour dans son cœur," la princesse de Cadignan "pour la première fois de sa vie souffrait dans son cœur et suait dans sa robe." 38 Les grandes amoureuses de Balzac ont toujours les mains suantes, le front humide: "chez elles, le désir tout naturellement exsude."

Ainsi la résolution de l'intrigue évoquée au commencement de l'histoire est résolue. En effet, ce qui préoccupe la princesse de Cadignan, se confessant à la marquise d'Espard, c'est qu'elle n'a "jamais rencontré l'amour en essayant d'aimer. [...] Je suis poursuivie dans ma retraite par un regret affreux: je me suis amusée, mais je n'ai jamais aimé [...] et nous sommes, me semble-t-il encore assez vivantes pour prendre une revanche."39 Bien au contraire, une main glacée sera le signe d'un état sans vie. La description du couvent que l'on trouve dans $\underline{L a}$ Duchesse de Langeais se caractérise par une absence de mouvement, de chaleur, de clarté, couleurs sombres: le couvent

38 Les Secrets de la princesse de Cadignan, VI, 64

39 Ibid, 19 
est noir, brun et gris ( $V, 127)$. Aussi, Armand de Montriveau observe que "la main glacée d'une femme, celle de la Supérieure sans doute, tenait encore le rideau" ( $V, 138)$. II faut remarquer que c'est ici l'auteur qui projette en fait sa propre perception puisque Montriveau n'a pas touché la main de la Supérieure.

Quant aux lèvres elles soutiennent, toujours à l'intérieur de cette thématique, une vitalité effusive, un trait de transparence ou de provocation. On sait que la psychanalyse les marque comme une zone érogène décisive. A travers leur finesse on devine en effet la pulsion, le frémissement du sang; parfois même l'on peut l'y toucher, l'y goûter: c'en est ainsi dans le baiser. Le rouge de leur pulpe signifie donc la manière non douteuse d'une certaine puissance à exister. De larges lèvres seront ainsi presque toujours chez Balzac des indices de bonté. Et cela d'autant mieux qu'à leur charnu, à leur richesse sanguine sous-jacente s'ajoutera la subtilité de leurs inflexions. Le juge Popinot possède ainsi "une bouche sur les lèvres de laquelle respirait une bonté divine. C'était de bonnes lèvres rouges, à mille plis, sinueuses, mouvantes, dans lesquelles la nature avait exprimée de beaux sentiments." 40

Mais c'est dans la totalité de l'étendue charnelle qu'il faut voir comment l'ardeur vitale se dispose, s'équilibre, se normalise. C'est là qu'apparaîtront les diverses figures, positives ou négatives, heureuses ou malheureuses, offertes ou

40 L'Interdiction, III, 21 
refusées, de son économie. Fils conducteurs de force, les nerfs peuvent en effet chauffer: ainsi la marquise d'Espard, toujours agitée d'intrigues et de passions changeantes, était, nous dit Balzac, “une femme d'un tempérament sec et nerveux; sans son régime, son teint eût pris la couleur rougeâtre que donne un constant échauffement." 41

Et que dire de la duchesse de Langeais en qui, nous le savons, Balzac a sans doute fixé les souvenirs d'une frustration très personnelle?* Pourquoi laisse t-elle s'éteindre la flamme après l'entrevue au couvent? Elle avait le choix entre le retour à la passion et la mort en Dieu. C'est son refus ultime qui la perd. A quoi son obstinée résistance est-elle due? Sans doute à un défaut d'onction, qui s'exprime dans le visage par les motifs de l'étroit, de l'allongé (lui-même traduit en aristocratique, en archaïque):

Sa figure un peu trop longue avait de la grâce, quelque chose de fin, de menu, qui rappelait les figures du MoyenAge. Son teint était pâle, largement rosé. Tout en elle péchait pour ainsi dire par un excès de délicatesse. 42

41 Ibid, 45

* Cf. Fiction et Vérité, I, XXIX. La duchesse de Langeais est reconnue comme étant le reflet de la marquise de çastrie, maîtresse décevante de Balzac.

42 La Duchesse de Langeais, V, 153 
L'élongation des formes n'est-elle pas ici synonyme de non-largeur humorale, disons même de non-largesse; et cet excès final ne nous renvoie qu'à une carence de la vitalité. Balzac récuse la conception suivant laquelle la beauté idéale serait fondée sur l'absolue régularité des lignes. La beauté vraiment merveilleuse de Natalie Evangelista "venait d'une excessive régularité des traits en harmonie avec les proportions de la tête et du corps. Cette perfection est de mauvaise augure pour l'esprit."43

Dans les figures d'une physiologie heureuse nous rencontrerons par opposition la fluidité. Pour premier visage elle aura celui de pulposité devinée, du mœlleux. En opposition aux maigreurs et sécheresses des femmes négatives, la femme désirable offre tout d'abord à l'œil, puis à la prise imaginée, la large étendue d'une chair tendre. C'est par la silhouette que Balzac traduit la qualité de l'être et non par la taille. D'autre part, si telle ou telle partie du corps a plus d'importance qu'un autre, ceci est à mettre en parallèle avec le principe fondamental de la vie selon Balzac, que nous avons examiné en première partie; ce principe fondamental est qu'il n'y a pas deux âmes semblables parce qu'aucune n'a des idées ou sensations semblables et qu'en conséquence l'originalité de l'âme doit imprimer son caractère au corps. De là l'harmonie des proportions est à considérer comme une loi des rapports entre 
le corps et l'âme, comme une loi d'exacte représentation par les formes des qualités singulières de l'être intérieur.

Dans le portrait des femmes aristocratiques que fait Balzac, on peut ainsi remarquer qu'il adopte l'esthétique romantique, contre l'idéal classique, pour laquelle le charme d'un visage ne réside pas dans la la parfaite régularité des traits qui ne signifie rien qu'absence de vie, mais naît du "caractère" que mettent en relief des lignes au modelé accusé par une pensée active ou une passion forte. Leurs valeurs morales ainsi revélées par la vérité extérieure, se marquent généralement d'insensibilité. La force exulte dans le caractère et l'intelligence. On trouve en Diane "une vigueur d'âme, une lucidité d'aperçus, une promptitude de décision, une insouciance ou plutôt un parti-pris sur certaines choses dont s'effraierait un homme."45 Diane avait

la faculté de séparer d'elle-même" et de renoncer à son gré à ses frivoles mondanités pour faire face à temps voulu, avec un sang-froid viril, aux nécessités de la vie. Elle avait le pouvoir de contempler le désastre à quelques pas, au lieu de se laisser enterrer dessous. C'était certes grand mais horrible dans une femme. [...] Elle avait contemplé le danger dans toute son étendue, la possibilité d'une chute épouvantable. [...] Ces divers

44 Béatrix, II, 195.

45 Les Secrets de La princesse de Cadignan, VI, 39 
moyens, cette catastrophe, tout avait été délibéré froidement, avec calme, sans trépidation. ${ }^{46}$

Diane n'éprouve plus de vertige devant la chute sociale; bien au contraire, elle expose le portrait de Charles $X$

par de Mirbel, sous lequel était gravé ses mots: donné par le roi. Sur la table brillait un album du plus haut prix, qu'aucune des bourgeoises qui trônent actuellement dans notre société industrielle et tracassière n'oserait étaler. Cette audace peignait admirablement la femme. ${ }^{47}$

Lorsque ce type de femme se trouve alors confrontée au sentiment d'amour, sa sensibilité et donc le thème de la sensibilité féminine, s'exprime tantôt ironiquement, tantôt sur un mode pathétique. "Qu'importe le nombre de jours" s'écrit I'héroïne de La Peau de chagrin, "si, dans une nuit nous avons épuisé toute une vie de paix et d'amour." De même, mêlant l'ironie et la sympathie, il déclare dans Ferragus que rien n'est plus contraire à la nature des femmes qu'un amour tranquille et parfait: loin de là elles ne désirent que des émotions. La Duchesse de Langeais est en effet condamnée par la présence d'un certain masochisme dans la mentalité d'un sexe qui est capable de "regretter les émotions de la peur" tant une femme 
peut être "avide de sensations extrêmes." Peut-on ici se permettre de croire que ce sont les échecs sentimentaux de Balzac, son état moral dû à la perte irréparable de Mme de Berny, ces espoirs perdus en ce qui concerne Mme Hanska, le sentiment d'une solitude impossible à briser, qui le poussent à se faire une conception désabusée de la femme en qui il voit dès lors un être trompeur ou du moins décevant? Car les solutions qu'il offrent aux hommes pour attendrir des chairs si étrangères sont pour le moins on ne peut plus violente. Dans Le Contrat de mariage, de Marsay explique à Paul de Mannerville, mauvais élève, que l'homme doit se servir d'un "poignet de fer," pour rendre son épouse "ductile." "La crainte inspirée par l'amour," affirme t-il, "est un instrument infaillible pour manier l'esprit d'une femme." La seule tactique pour séduire l'acier, la glace, le roc, est de leur opposer un autre acier, une autre glace, un autre roc. Face à de telles femmes le moindre signe de faiblesse entraînerait un échec sans remède. "Pour faire jaillir l'amour dans une nature siliceuse, il faut, nous dit Balzac, un homme de fer." 48 Et ailleurs: "Pour fondre, pour vivifier ces cailloux,"ce sont “les femmes froides, frêles, dures et minces"qu'ont évoqué toutes les analyses antérieures, il faudra des "coups de foudre." Et le mot foudre doit être pris dans tous ses sens, jusqu'au premier. Seule l'explosion d'une violence ou l'éclatement d'un feu auront quelques chances de briser puis de liquéfier, donc

48 La Duchesse de Langeais, V, 216 
d'animer la pierre ou le métal, de faire capituler la femme froide. C'est ce dont témoigne du moins l'aventure de la duchesse de Langeais: Montriveau menace Mme Langeais de la marquer au visage en la brûlant, ce qui casse enfin sa résistance:

Deux de mes amis font rougir en ce moment une croix dont voici le modèle [...] Toujours et plus que jamais, c'est alors que l'état de la flamme se manifeste dans ce tourbillon de rouges ("lueurs rougeâtres") et d'images de flammes dans la bouche de la duchesse, et de façon sincère: "Ah! mon Armand, marque, marque, marque vite ta créature [...] quand tu auras une âme serve qui te portera ton chiffre rouge, eh! bien, tu seras à jamais à moi. [...] Entrez vite, et tous, mon front brûle plus que votre fer! 48

Quand à l'âge de trente ans Balzac publie la Physiologie du mariage, et chacun sait combien cette œuvre est pleine d'esprit mais aussi insolente, sans doute Balzac avait été inspiré par les vicissitudes de sa vie qui le mettaient si proche de l'adultère. Il déclare: "la destinée de la femme et sa seule gloire sont de faire battre le cœur des hommes. La femme est une propriété que I'on acquiert par contrat; [...] elle n'est, à proprement parler, qu'une annexe de l'homme." Mais fort heureusement Balzac se

49 Splendeurs et misère des courtisanes, 84. 
contredit avec éloquence quand il ne s'agit plus de fournir préceptes et maximes, et cela permet à l'écrivain qu'il est de modeler des femmes bien vivantes, capables de sentiments, de désirs et d'émotions chargées d'intelligence (cf. Honorine). Mais que penser de ces mots: "Toutes les femmes aimantes et dévouées inventent la réclusion, l'incognito, la vie de la perle au fond de la mer?"49 D'autre part, c'est de façon frappante que la Duchesse de Langeais incarne certains "dangers de l'amour," terme employé par Balzac lui-même. Dans l'espoir de posséder cette femme trop désirée, Montriveau met le pied dans un "enfer" où il souffre de "fièvre amoureuse."

L'élément de cruauté est souligné dans la suite de ce drame de l'amour frustré. Montriveau veut se venger en marquant la duchesse avec un fer rougi, trait qui indique la présence d'un certain sadisme. II est en effet certain que la liaison malheureuse avec la marquise de Castries a jeté de nombreux troubles sur ce roman qui montre l'amour comme une passion cruelle. Mais comme l'a montré P.-G. Castex dans son édition de la Duchesse de Langeais, ce serait simplifier à l'excès que de voir cette histoire comme un acte de vengeance contre Madame de Castries qui s'était refusée au romancier. Avant d'analyser le paradoxe tragique des destins de ces femmes, il est intéressant de noter qu'une préface au Père Goriot contient une argumentation ironique dans laquelle 
l'écrivain entreprend plaisamment de démontrer, chiffres à l'appui, qu'il avait créé plus de femmes vertueuses que de coupables. 


\section{CHAPITRE IV}

\section{Paradoxe Tragique}

Nous avons pu nous rendre compte dans le chapitre précédent que dans la représentation de la femme, et de toute manifestation vivante, la façon dont respirait les corps et plus généralement, la façon dont s'exprimait la Pensée sur le plan physique, prend une place considérable dans le travail du romancier. II lui voue un intérêt particulier, par ses descriptions massives, car Balzac est convaincu que le physique est à la fois source (i.e. l'amour en tant que besoin physique) et épiphénomène, et qu'il fournit le seul moyen de reproduire et de communiquer les événements qui se déroulent au niveau de la Pensée, événements sociaux ou événements moraux. ${ }^{1}$ Le plan physique est, par conséquent, la base qui porte les deux autres (plan social et plan moral), mais il n'est n'est pas la scène véritable sur laquelle se jouent les drames.

Du plan social dépend l'individu, et par son existence sur le plan social, il se trouve situé, historiquement, par le lieu et par le moment, en dépendance d'une situation collective. Par "sa nature sociale" l'individu dépend des autres, de même qu'il est responsable devant eux de ses actes. Les relations qu'il peut avoir sont des relations de forces, des relations de propriété et d'argent. Les ambitions et idéalismes, rivalités et infidélités, passions et vices, ne sont vécus que dans la mesure où ils sont

1 Chapitre sur Les Sphères dans Le Monde de La Comédie humaine, par Gaston Zélicourt, p259 
suscités par l'existence sociale. Et la femme idéale, selon Balzac, ne doit-elle pas posséder d'ailleurs cette perfection sociale?

Quel homme, en quelque rang que le sort l'ait placé, n'a pas senti dans son âme une jouissance indéfinissable en rencontrant, chez une femme qu'il choisit, même rêveusement, pour sienne, les triples perfections, morales, physiques et sociales, qui lui permettent de toujours voir en elle tous les souhaits accomplis?2

Et c'est un engrenage puisque, comme il a été dit dans notre premier chapitre sur l'existence et l'essence humaine, l'Homme appartient à une "espèce sociale." Ce qui ne signifie en rien que les phénomènes spirituels qui naissent de là s'en trouvent amoindris, ils sont tout aussi forts.

Le drame moral est le sort donné à la personnalité: sa cohérence harmonieuse ou sa dispersion devant les appels sociaux, son enrichissement et l'épanouissement de ses virtualités sentimentales ou intellectuelles ou la déperdition de ses possibilités.

La liaison entre Paul et Nathalie, dans Le Contrat de mariage, est un amour et une relation sociale, financière et juridique:

II se passa une double scène : au coin de la cheminée du grand salon, une scène d'amour où la vie paraissait riante 
et joyeuse; dans l'autre pièce, une scène grave et sombre, ou l'intérêt mis à nu jouait par avance le rôle qu'il joue sous les apparences fleuries de la vie. ${ }^{3}$

Lorsque l'amour et l'amitié ne passent pas par l'expression sociale, elles sont pures harmonies psychiques; et ce que ressent la duchesse de Langeais semble bien correspondre, bien au contraire, à un jeu social puis à un sentiment forcé par la main de fer de I'homme en question; sa passion revêt un manteau épais d'hypocrisie, aussi et surtout cousu du fil de l'orgueil. Par conséquent, si le pardon prévaut dans la conception chrétienne, l'orgueil, le mensonge, l'absence de sincérité et la vanité conduisent à un destin tragique. Quant à l'expérience sociale, il est clair que dans le petit roman qui porte son nom, elle est le produit typique de son milieu: parfaite, technicienne consommée dans son domaine, mais absolument froide et moralement inexistante:

Elle était coquette, aimable, séduisante jusqu'à la fin de la fête, du bal, de la soirée; puis le rideau tombé, elle se trouvait seule, froide, insouciante [...]. Elle se disait: - Je suis aimée, il m'aime! Cette certitude lui suffisait. Semblable à l'avare satisfait de savoir que ses caprices

${ }^{3}$ Le Contrat de mariage, III, 116 
peuvent être exaucés, elle n'allait peut-être même plus jusqu'au désir. 4

Elle allait donc exercer son art de comédienne devant cet homme exceptionnel: noble, il possédait une grande élévation d'esprit; militaire, il savait donner et avait prouvé son dévouement; et, comme pour achever le portrait jusqu'à la perfection, il était aussi homme de science. Le numéro de routine exécuté par la duchesse provoque chez lui une émotion réelle, une véritable extase qui le rend presqu'inexistante en elle-même. La duchesse de Langeais s'est alors transformée en une réalité dans le drame moral. Sa réaction est réelle et excessivement forte, de telle sorte que l'orage qui en résulte brise la carapace d'amour-propre et de vanité qui avait enfermé la femme du monde. Naît en elle une nouvelle femme, une femme vivante qui, avant de mourir, aura vécu une vie morale à une hauteur spirituelle jusque là inconnue d'elle. II est vrai, cependant, que si Balzac semble faire un don inégalable, il semble ainsi le reprendre aussitôt, car cela ne va pas plus loin. La qualité métaphysique et religieuse apparaît plutôt de mauvais aloi et pour Montriveau l'histoire n'aura pas non plus de lendemain. Quel est le drame de la duchesse? Son drame est celui du heurt entre la coquetterie et l'amour. Le crime de la coquette est d'avoir à la fois appelé et refusé l'amour: "Mais

${ }^{4}$ La Duchesse de Langeais, V, 159 
vous souvenez-vous qu'un jour vous avez appelé l'amour [...] vous vous êtes jouée de cet amour, vous avez commis un crime." 5 En termes chrétiens, son châtiment sera d'éprouver trop tard l'amour vrai, sa grandeur sera de rompre avec les vanités et de s'imposer retraite derrière la clôture d'un couvent de carmélites.

Mais à mon avis (et les critiques semblent oublier ce détail sur lequel Balzac n'insiste pas non plus dans le roman), son drame, vient de plus loin. Son mariage n'avait été qu'un mariage de convention, qui avait permis au duc de Langeais, tout en sauvegardant les apparences, de l'offenser gravement en l'abandonnant à l'âge de vingt-deux ans. Il y a pire encore: sa nuit de noces ne lui a rien apporté. Ainsi l'union avec un homme n'éveille en elle aucune image et pour cette raison vraie, Antoinette a pu devenir une froide coquette. Lorsqu'elle rencontre le général de Montriveau, homme à cœur et face de lion, l'occasion est belle pour déployer sa coquetterie. Par des alternatives d'encouragements et de dédains, elle irrite cet homme prestigieux pendant des mois . Montriveau s'exaspère et finit par s'éloigner bien qu'il soit violemment épris de cette femme séduisante, une des reines de Paris. C'est ce départ qui fait prendre conscience à la duchesse de la place occupée par Montriveau: le passage progressif de la comédie à la sincérité s'effectue. Pendant huit jours elle l'attend en vain. Ce n'est pas 
l'amour mais c'en est le prélude. A l'attente se mêle une crainte: elle est persuadée que cet homme à cou de taureau est capable d'une vengeance inouïe. En effet, Montriveau la fait enlever. II lui reproche de s'être refusée après avoir provoqué l'amour. Pour la punir de ce crime, il va lui appliquer la marque infamante des forçats. Mais elle accepte d'être défigurée, s'appuyant sur une illumination de l'amour: par apposition de cette marque, Armand proclame au monde qu'il s'approprie Antoinette et, dès lors, il n'aura plus le droit de l'abandonner. N'est-ce pas là, en fait, toute l'attente de la duchesse? Car encore une fois, l'attitude de la coquette, typique de l'aristocrate du Faubourg Saint-Germain, peut sans doute trouver son explication dans son contexte historique. La valeur de la femme, aussi bien morale que sociale, n'était même pas proche d'atteindre le centième de ce que l'on accordait aux hommes; leurs qualités physiques préfiguraient, et la place faite à leur esprit n'était en vérité qu'illusoire. C'était en pratiquant leur art de comédienne, qu'elles rendaient bien aux hommes leur attitude méprisante. Pouvons-nous, par conséquent, peut-être expliquer l'attitude de la duchesse par une peur inconsciente d'être la cible du jeu des hommes? Touché par son acceptation d'être ainsi marquée, ce qui manifeste I'amour vrai, Armand renonce à I'appliquer; néammoins, pour cet homme fort, tout est fini et il reconduit Antoinette sans lui dire adieu. C'est alors qu'Antoinette endure toutes les souffrances de l'amour méconnu. Jour après jour elle 
écrit à Armand sans obtenir de réponse. Cependant une duchesse ne peut pas avoir dit à un homme : je suis à toi! Ou elle doit être aimée ou elle abdique son rôle social. Aussi, par une lettre d'amour fou et d'adieu, elle lui fixe un dernier rendez-vous avant de quitter le monde. Par un hasard malencontreux, Montriveau manque le rendez-vous et la duchesse s'exile sans que l'on puisse retrouver sa trace.

Lorsque les deux amants se retrouvent à la grille des carmélites, Montriveau fera des déclarations d'amour fou à celle qui est devenue Sœur Thérèse; de cette femme ne semble plus rester que l'âme, "une âme" dépouillée de tous liens humains" qui "soupirait après le long suicide accompli dans le sein de Dieu."6 Et ceci se retrouve bien dans sa représentation extérieure, elle est amaigrie et ne semble plus avoir d'âge. Aussi est-elle presqu'entièrement dépeinte par sa voix, sa voix qui avait gardé "son timbre clair." De ce qui fut, jadis, le diamant des salons parisiens ne reste que la voix:

Cette voix, légèrement altérée par un tremblement qui lui donnait toutes les grâces que prête aux jeunes filles leur timidité pudique, tranchant sur la masse du chant, comme celle d'une prima donna sur l'harmonie d'un finale. [...] Elle semblait dire à son amant: _ [...] Tu m'entendras, mon âme t'enveloppera [...] tu ne me verras pas. [...] Cette voix

6 Ibid, V, 123 
connue vibra [...] cette voix si riche continuait à déployer toutes ses calineries $[\ldots]^{7}$

Bien qu'elle fut toute dévouée à Dieu, Montriveau est illusionné par le jeu des orgues et croira sentir qu'elle aime encore. En 1822, le duc de Langeais étant mort, sa femme devient libre et le pape peut casser les vœux de la Sœur Thérèse. Aussi Montriveau et et des amis dévoués (Les Treize) entreprennent de l'arracher à son cloître. Lorsqu'ils y pénètrent, c'est le moment d'un office des morts, qui, par un coup du sort, est l'office même de la Sœur Thérèse. Dieu a fait les dénouements.

L'une des explications apportée par la plupart des critiques est que Balzac n'hésite pas à reprocher à l'aristocratie de la Restauration d'être devenue un monde sans force pour s'être coupé des réalités nouvelles : quand une révolution est passée dans les mœurs, il faut savoir s'y adapter. La duchesse de Langeais est dans ce monde pleine de sentiments élevés, mais manquant d'une pensée qui les coordonne et n'ayant pas su apprécier le changement des temps. Aussi, envisagé sur le plan individuel et moral, l'accès à une Pensée plus complexe et plus civilisée semble entraîner, par la même occasion, une tentation à la démesure. Pourquoi est-ce-que la duchesse s'entête à refuser un amour qu'elle sait alors sincère? 
Si l'on examine maintenant le drame de Diane de Maufrigneuse, dans Les Secrets de la princesse de Cadignan, il est évidemment moindre. Malgré le fait qu'elle ait à vivre dans un éternel mensonge, elle finira heureuse avec d'Artez dans un mélange de sagesse et de bonheur tranquille. Car, elle qui n'avait trouvé jusque là que des hommes "petits, mesquins, superficiels," des hommes qui n'avaient pas "causé la plus légère surprise, ils étaient sans innocence, sans grandeur, sans délicatesse," en avait enfin trouvé un qui l'eut "imposé."

Dans un salon aristocratique se joue aussi le drame de madame de Beauséant. La tragédie de madame de Beauséant est celle du délaissement, l'abandon-affront. Le dégoût qu'elle en ressent versera un acide corrosif sur les conseils donnés à Rastignac dans Le Père Goriot.

Le prix à payer pour l'ascension vers une sorte d'apogée de l'activité de la Pensée est lourd, et ceci est d'autant plus vrai lorsque l'on monte dans le monde balzacien. Quand on arrive au niveau parisien, la gamme des passions possibles s'étend, s'enrichissant d'un éventail de passions ou de vices plus ample que celui de la province, qui ne connaît, grosso modo, que l'avarice ou la haine ou la vengeance. Dans le milieu parisien s'y ajoutent l'érotisme, les passions adultères, les rivalités dans un luxe qui dépassent les moyens dont chacun dispose, la gastronomie et surtout une agitation incessante inspirée par cette chasse à l'or et aux plaisirs qui est le refrain de la grande vision d'ensemble; mais le prix à payer est cher. 
Balzac était persuadé que le XIXe siècle français allait faire faire un pas gigantesque à l'Humanité dans un progrès réel, non pas en inventions, mais en prise de conscience. C'est là le sens de l'Avant-Propos de 1842:

Je ne partage point la croyance à un progrès indéfini, quant aux sociétés: je crois au progrès de l'homme sur luimême. Ceux qui veulent apercevoir chez moi l'intention de considérer I'homme comme une créature finie se trompent donc étrangement. 8

Balzac pouvaient voir en ces souffrances des femmes une source possible de grandeurs morales et spirituelles supérieures; cependant, il semblait plus urgent de "réaliser des réformes qui permettraient de redresser les torts de la Société sur ce point."

Le petit roman intitulé Le Contrat de mariage, destiné à "retracer la grande comédie qui précède toute vie conjugale" conclut ainsi:

Dans la plupart des familles, la constitution des dots et des donations à faire au contrat de mariage engendre ainsi des hostilités primitives, soulevées par l'amour-propre, 
par la lésion de quelques sentiments, par le regret des sacrifices et par l'envie de les diminuer. Ne faut-il pas un vainqueur et un vaincu, lorsqu'il s'élève une difficulté??

Balzac, a certainement voulu donner une certaine liberté à la femme mais avec une restriction de taille: il a tendance à lui faire choisir l'abnégation, comme il était commun au XIXème siècle. Et ceci est une façon de reprendre ce qu'on a donné. Le roman intitulé Le Lys dans la vallée en est un exemple parmi tant d'autres; il semble bien y avoir une sorte de divination de la femme, mais cette apothéose est précédée d'un sacrifice important puisque Mme de Morsauf est "immolée au devoir." Sans doute est-ce grâce à tout le sentimentalisme que Balzac déploie, et ce qui n'était pas non plus pour déplaire au goût du temps, que son paternalisme et quelques tendances que l'on peut avec précaution appeler misogynes.

"Le droit d'aimer" était l'une des exigences principales formulées par les féministes de 1830. Et c'était là une revendication qui rejoignait, logiquement, le romantisme. Les féministes de cette époque soutenaient cette théorie selon laquelle la femme mérite de tenir un rang élevé dans la société car elle a toutes les qualités affectives et intuitives pour $y$ accéder. II fallait donc pouvoir ressentir l'amour sans perdre sa dignité, et pour cela il fallait que les femmes arrachent aux

9 III, 116 
hommes cette liberté sexuelle qu'ils leur refusaient. Balzac, sur ce point, avait des théories apparemment contradictoires. La Physiologie du mariage en est un bel exemple; l'on y trouve des éléments qui nous permettent de qualifier Balzac de misogyne et de féministe. La première Méditation de l'Etude affirmait que "la vie est dans la passion, et qu'aucune passion ne résiste au mariage."10 Car, rappelons-le, dans la société de l'époque, tout jugement porté sur la condition féminine supposait un examen du système conjugal. Ainsi il continue: "Que le mariage est une institution nécessaire au maintien des sociétés, mais qu'il est contraire aux lois de la nature."

Le problème est donc un conflit entre la passion et la raison, la nature et la société; ce qui nous ramène aux problèmes évoqués dans notre premier chapitre, à savoir la difficulté à définir les positions philosophiques de Balzac et d'où le paradoxe que l'on trouve tout au long de l'œuvre. Un examen des différentes attitudes adoptées par l'auteur montre l'existence d'un conflit entre deux tendances qui cohabitent en lui et qui s'expriment à tour de rôle. Justifier l'amour passionné, excuser l'adultère, ou se montrer sévère en accord avec la morale chrétienne, Balzac se montre généralement sympathique aux victimes d'un premier amour mais son jugement peut aussi accuser une sévérité extrême; comment

10 La Physiologie du mariage, X, 603 
peut-on se faire l'avocat du recours à la force et de la violence dans ce même contexte moral?

Les femmes mariées, comme Mme de Beauséant, sont l'objet de cette hésitation à choisir entre les impératifs de la société et ceux de la nature. Abandonnée par un amant, elle vit à l'écart du monde, dans un coin de province où elle se cache. Reniée par son mari, elle est l'objet des désapprobations des gens de la région qui le montrent bien à travers la froideur qu'ils lui réservent. Mais Gaston de Nueil admire la fierté de la femme bannie et réussit enfin à lui communiquer l'amour qu'il a conçu pour elle. Ils connaissent neuf ans de bonheur mais ils ne peuvent s'unir légalement. Le jour arrive où la passion ressentie par le héros s'attiédie au point qu'il accepte un mariage d'intérêt arrangé par sa famille. Déçu par la froideur de cette nouvelle union, Gaston essaie de se rapprocher de son ancienne maîtresse. Fièrement repoussé par elle, il se tue. N'est-ce pas là toute la force et la beauté qu'un cœur de femme peut conférer à l'amour, "germe indestructible" qui donne naissance à l'amour? Aussi la nouvelle contient-elle un véritable plaidoyer en faveur du droit d'aimer. Cette femme qui est l'objet d'une admiration certaine, dépeinte avec tant de sympathie, commet deux fois l'adultère; et l'auteur y montre bien peu de sévérité. Mme de Beauséant, justifiant son amour, commente sur ce qui oppose les convenances aux désirs du cœur: 
Je n'ai pas eu la haute vertu sociale d'appartenir à un homme que je n'aimais pas. J'ai brisé, malgré les lois, les liens du mariage: c'était un tort, un crime, tout ce que vous voudrez; mais pour moi cet état équivalait à la mort. J'ai voulu vivre. N'est-ce pas une loi de notre nature que d'être heureuse? 11

La seule loi qui vaille quelque chose est celle de l'amour, et c'est ce qu'elle explique dans une lettre à Gaston: "toute la femme est dans ce sentiment, qui sanctifie tout." Lorsque Mme de Beauséant et ses sœurs invoquent leur droit au bonheur, parlant de cette "loi de leur nature" qui ordonnent aux femmes d'aimer et d'être heureuse, Balzac est loin de refuser sa sympathie à cette protestation; la preuve en est claire quand, à la fin de l'histoire, il développe encore cette loi du cœur, accusant le héros d'avoir abandonné, pour une chimère sociale, sa "véritable épouse."12

La vie passionnée de Mme de Beauséant a une fin tragique, mais ce fait ne semble qu'en hausser l'attrait romanesque. La liberté coûte chère. Seules quelques femmes la poursuivent et le romancier se montre compréhensif envers celles qui ne craignent pas d'entrer en conflit avec la société.

Mais l'apologiste de la passion, ne l'oublions pas, se fait aussi défenseur de la morale chrétienne, ce qui le pousse aussi

11 La Femme abandonnée, II, 225

12 Ibid, II, 238 
à donner quelques leçons de sagesse. Pour cette raison même, l'amour coupable rencontré chez tant de personnages féminins revêt l'apparence d'une fatalité, souvent tragique. Mme de Morsauf, malgré toutes les circonstances qui pourraient la faire s'enfuir devant le devoir, parvient à assurer l'avenir d'une famille vieille et respectable. Elle a un mari décevant, à moitié fou; elle aime et est aimée. Mais la vertu de cette femme lui donne la force de sacrifier son amour à son devoir conjugal et familial. Aussi, elle devient le véritable chef de famille, la directrice de sa propriété matérielle et morale.

Balzac excuse dans ce cas l'adultère car sans doute le personnage en question est aussi capable du plus haut sacrifice pour sauver sa famille. En effet, ce que Balzac avait remarqué, c'était que l'esprit moderne, avec son individualisme moderne, détruisait progressivement l'esprit de la famille. Balzac méprisait les mœurs de son époque qu'il considérait comme "dépravées."

Lorsqu'il refuse dans le cas de la duchesse de Langeais d'admirer sa passion, Balzac montre en fait que cette passion n'est pour lui qu'une singulière hypocrisie. En effet, le romancier a toujours exposé son admiration pour les passions véritables; et pour cette raison même qu'il ne croit pas en la duchesse qui ne fit, en fait, que faiblir sous le joug des fers de Montriveau, sa passion se tranforme alors en une triste passion. La faute commise par la duchesse se présente à mes yeux comme ceci: elle devient, en fait, "responsable" de son passé, un 
passé dont elle ne peut, par conséquent être absoute. Et par la même occasion, Balzac semble adopter des attitudes qui ressemblent fort à une justification des fautes commises par les femmes.

D'autre part, si l'on pense au fait biographique, il me semble que Balzac s'adresse directement à Mme de Castries, faisant, en quelque sorte, son procès, mais aussi le procès de toute une caste qu'il répudiait sans doute au moment de cette grande déception:

A mesure que l'on monte en haut de la Société, il s'y trouve autant de boue qu'il y en a par le bas; seulement elle s'y durcit et se dore. Oui, pour rencontrer la perfection dans l'ignoble, il faut une belle éducation, un grand nom, une jolie femme, une duchesse. Pour sombrer au dessous de tout, il fallait être au dessus de tout. Je vous dis mal ce que je pense, je souffre encore trop des blessures que vous m'avez faites; mais ne croyez pas que je me plaigne! Non. Mes paroles ne sont l'expression d'aucune espérance personnelle, et ne contiennent aucune amertume. Sachez-le bien, madame, je vous pardonne. ${ }^{13}$

La réponse à nos questions semblent ainsi trouver une réponse en accord avec la théorie avancée par Pascal sur la 
grâce. Le monde de Balzac est définivement commandé par les forces divines; tout comme il croyait en la monarchie, car le roi était pour lui une représentation terrestre de Dieu: un roi divin. Ainsi, malgré la reconnaissance des qualités animales, naturelles de l'homme, qui en font un être de passions, il semble que la dimension divine l'emporte en fin de compte. Cette constatation d'une suprématie du monde divin fait que le monde physique, moral, et social s'en trouvera inévitablement fortement influencé. S'appuyant sur de telles théories, Balzac paraît aussi se réserver le droit de jugement ultime sur les passions, et donc sur "le droit d'aimer" de ses personnages qui nourrissent de telles ambitions.

D'autre part, ce qui peut-être fait la différence entre le jugement porté sur la duchesse de Langeais et Mme de Morsauf, ainsi que Mme de Maufrigneuse, c'est l'âge. Balzac ne croyait pas qu'une jeune femme puisse apporter le bonheur à un homme, et là se trouve la conclusion à La Duchesse de Langeais: "Il n'y a que le dernier amour d'une femme qui satisfasse le premier amour d'un homme" (V, 254). 


\section{CONCLUSION}

La question véritable n'est pas de placer Balzac dans un courant de pensée qui appartenait bien évidemment en partie à son époque; il est inutile de débattre de sa misogynie ou de son féminisme puisqu'il nous est apparu clairement que les deux tendances s'insèrent dans son œuvre. Que le ton employé soit pathétique ou ironique, que se soit dans l'évolution d'un même personnage ou dans la résolution d'un même roman, ces tendances divergent mais sans être exclusives l'une de l'autre.

Balzac croyait fermement en l'existence d'un monde divin au dessus de toute autre sphère. Le christianisme était pour lui, rappelons-le, seul capable de redresser l'Ordre Social; un Ordre Social qu'il ne cesse d'ailleurs de contester et de questionner lui-même. L'Etat Social définit tout être: il est la Nature plus la Société; tout être apparaissant logiquement tout d'abord dans sa nature animale. De ce fait deux forces contradictoires vont se heurter, et dans toute résolution du roman balzacien l'on peut y percevoir le châtiment chrétien, ou bien son pardon.

Cependant, même quand Balzac s'exprime en moraliste catholique, il reste un fond de non-conformisme et une conception axée sur l'exception. L'amour coupable qu'il a peint avec indulgence chez tant de personnages féminins se colore souvent d'une fatalité tragique.

Balzac a su dépeindre, de façon éloquente, les manifestations de la vie. Les femmes l'ont, de toute évidence, 
passionné; les plus hautes sphères de la société l'ont captivé. La femme aristocrate, en plus d'être l'objet de son admiration, fut l'objet de nombreux romans et de nombreuses "scènes," avec une ressemblance fidèle à la réalité du temps. Sainte Beuve qui était de son époque, et du même monde, nous garantit la ressemblance: "qui mieux que lui, a peint les vieux et les belles de l'Empire? Qui surtout a plus délicieusement touché les duchesses et les vicomtesses de la fin de la Restauration?"1 Elle pouvait, grâce à Balzac, se voir en tant que réel sujet, ayant un droit véritable à la passion. Mais si les jeux sociaux se faisaient plus forts que les sentiments, que la carapace de la coquette ne puisse se briser, le tableau s'assombrît.

Balzac était avant tout un grand observateur de son temps et de la nature humaine; peut-être y va-t-il aussi du fait que la nature offre elle-même tant de facettes à l'individu et tant de destinées divergentes, pour des raisons parfois inexplicables, que Balzac paraît se contredire dans ses attitudes. Mais, comme l'homme est une créature en perpétuelle évolution, selon Balzac, en progrès constant, le romancier lui-même a pu évoluer dans son jugement des hommes et des femmes, et par conséquent dans leurs représentations. Que ce soit dans leur chair ou dans leur âme, le tableau est éloquent et vrai.

1 Citation recueillie dans le chapitre sur la moralité de l'œuvre de Balzac dans Honoré de Balzac, par Ferdinand Brunetière. 


\section{Works Consulted}

Balzac, Honoré de. La Comédie humaine. Ed., Pierre-Georges Castex. Tomes I-X. Paris: Pléiade,1964.

Barbéris, Pierre. Le Monde de Balzac. Paris: Arthaud, 1973.

-------- Lecture du réel. Paris: Editions Sociales, 1973.

Barthes, Roland. S/Z. Paris: Editions du Seuil, 1970.

Bose, Mandakranta. Identity through Rebellion: A study of Women in Balzac, Dickens, and Saratchandra. Ed. R. C. Prasad and A. K. Sharma. New Delhi: Vikas, 1987. 273-83.

Brunetière, Ferdinand. Honoré de Balzac (1799-1850). Paris: Calmann-Lévy, 1906.

Castel-Carriga, G. "Une Aventure inconnue de la duchesse de Langeais." Revue des Deux Mondes, Jan. 151958.

Castries, duc de. "La duchesse de Langeais." Revue des Deux Mondes, Dec. 1977: 570-583

Chollet, Roland. "La Désespérance d'amour à la duchesse de Langeais." L'Année balzacienne. Paris: Ed. Garnier, 1965: 93-120

-------. Balzac journaliste: le tournant de 1830. Paris: Klincksieck, 1983.

David, G. "L'Idée de Bonheur dans La Comédie humaine." L'Année Balzacienne. Paris: Ed. Garnier, 1966.

Dupuis, Danielle. "Toilette feminine et réalisme balzacien." L'Année Balzacienne. Ed. Garnier, 1986. 115-38.

"La Poésie de la toilette féminine chez Balzac." L'AnnéeBalzacienne, vol 5. Paris: Ed. Garnier, 1985. 173-95. 
Festa-McCormick, Diana. "Sailing on the Balzacian Ocean of Women." Women in French Literature. Ed. Michel Guggenheim. Saratoga, CA: Anma Libri, 1988. 125-35.

Forest, Jean. Des Femmes de Balzac. Montréal: PU de Montréal, 1984.

------- . L'Aristocratie balzacienne. Paris: J. Corti, 1973.

Frangi, R. "Sur la duchesse de Langeais: un essai de lecture stylistique." L'Année balzacienne. Paris: Ed. Garnier, 1971: 236-52.

Fortassier, Rose. Les Mondains de La Comédie humaine. Paris: Klincksieck, 1974.

Frappier-Mazur, Lucienne. "Lecture d'un texte illisible: Autre étude de femme et le modèle de la conversation." MLN 98.4 (1983): 712-27.

Frary, Leon, comp. Selon Balzac: le cour humain. Paris: éditions Nelson, 1913.

Gancil, Farag. Le Costume et le caractère dans La Comédie humaine de Balzac. Le Caire: Les Livres de France, 1974.

Gauthier, Henri. L'Image de I'homme intérieur chez Balzac. Genève: Librairie Droz, 1984.

Hannosh, Michele. "La Femme, la ville, le réalisme: Fondements épistémologiques dans le Paris de Balzac." Romanic Review 82.2 (1991): 127-45.

Hokenson, Jan. "Male Feminists or the Breeches of Authority: The Monologue and its Relation to Ideologies of Gender." Romance Languages Annual 4 (1992): 70-7.

Lacroix, Paul. Les Femmes de Honoré de Balzac: types, caractères et portraits. 
Laparra, Camille. "L'Aristocratie dans La Comédie humaine de Balzac: ses pluralismes." The French Review 68.4 (March 1991): 603-13.

Lathers, Marie. "Modesty and the Artist's Model in Le Chefd'œuvre inconnu." Symposium: A Quartely Journal in Modern Foreign Literatures 46 (Spring 1992): 49-71.

Le Yaouanc, Moïse. "Sur l'Interdiction." L'Année balzacienne. Paris: Ed. Garnier, 1971: 253-60.

Mehta, Brinda Jagadish. "Representing Women in Balzac's Novels: An Aesthetics." Ann Arbor: DAl 49 (1989): 2243A.

Michel, Arlette and Jean Bessière, ed. "La Duchesse de Langeais et le romanesque balzacien." Figures féminines et roman. Paris: Presses Universitaires, 1982: 89-108.

----- Le Mariage chez Honoré de Balzac: amour et féminisme. Paris: Les Belles Lettres, 1978.

Moss, Martha. "The Masks of Men and Women in Balzac's Comédie humaine." The French Review 50: 3 (Feb 1977): 446-53.

Nesci, Catherine. La Femme mode d'emploi: Balzac, de La Physiologie du mariage à La Comédie humaine. Lexington, KY: French Forum, 1992.

Nik, Ninfa Verdi. "Women in Selected Fictional Works of Balzac and Galdos: A Comparative Study." Ann Arbor: DAl 46-3 (1985): 697A.

Nycrog, Per. La pensée de Balzac. Munksgaard - Copenhague: Scandinavian University Books, 1965.

Perren, Sara. "Balzac and Gender: Sexual Identity into Text in Three Novels by Honoré de Balzac." Ann Arbor: DAl 50 (1990): 3974A.

Perry, Katrina. "Absent Voices, Literary Bloodiness: A Study of Blood and its Relevance to Woman, Feminine Desire and 
98

Discourse in Balzac, Michelet, and Zola." DAl 51 (1991): 3098A.

Richard, Jean-Pierre. Etudes sur le romantisme. Paris: Editions du Seuil, 1970.

William, Robin. "'Deux vieux amateurs du beau sexe': Balzac and Hulot." Nottingham French Studies 24.1 (1985): 26-36.

Zélicourt, Gaston de. Le Monde de La Comédie humaine. Paris: Seghers, 1979.

Zweig, Stephan. Balzac, le roman de sa vie. Paris: Albin Michel, 1950 